\title{
Generalized refracted Lévy process and its application to exit problem
}

\author{
Kei Noba* and Kouji Yano*
}

\begin{abstract}
Generalizing Kyprianou-Loeffen's refracted Lévy processes, we define a new refracted Lévy process which is a Markov process whose positive and negative motions are Lévy processes different from each other. To construct it we utilize the excursion theory. We study its exit problem and the potential measures of the killed processes. We also discuss approximation problem.
\end{abstract}

\section{Introduction}

Exit problem of a real-valued stochastic process $Z=\left\{Z_{t}: t \geq 0\right\}$ is the problem to characterize the law of the first time of exiting an interval $[b, a]$ for $b<a$. In this paper, we are interested in the Laplace transform

$$
\mathbb{E}_{x}^{Z}\left(e^{-q \tau_{a}^{+}} ; \tau_{a}^{+}<\tau_{b}^{-}\right)
$$

for $q \geq 0$ and a starting point $x \in[b, a]$, where

$$
\tau_{a}^{+}=\inf \left\{t>0: Z_{t}>a\right\} \text { and } \tau_{b}^{-}=\inf \left\{t>0: Z_{t}<b\right\}
$$

When $Z$ is a spectrally negative Lévy process, it is well known that

$$
\mathbb{E}_{x}^{Z}\left(e^{-q \tau_{a}^{+}} ; \tau_{a}^{+}<\tau_{b}^{-}\right)=\frac{W_{Z}^{(q)}(x-b)}{W_{Z}^{(q)}(a-b)},
$$

where $W_{Z}^{(q)}$ is the $q$-scale function of $Z$.

Kyprianou and Loeffen [10] have studied the exit problem when $Z$ was a refracted Lévy process $U$, which was defined as the strong solution of the stochastic differential equation

$$
U_{t}-U_{0}=X_{t}-X_{0}+\alpha \int_{0}^{t} 1_{\left\{U_{s}<0\right\}} d s \quad t \geq 0
$$

*Department of Mathematics, Graduate School of Science, Kyoto University Sakyo-ku, Kyoto 6068502, Japan. Email: knoba@math.kyoto-u.ac.jp (K. Noba), kyano@math.kyoto-u.ac.jp (K. Yano). 
where the driving noise $X$ is a spectrally negative Lévy process and $\alpha$ is a positive constant. Define $Y_{t}=X_{t}+\alpha t$. Then the positive and negative motions of $U$ is given as

$$
U_{t}-U_{s}=\left\{\begin{array}{l}
X_{t}-X_{s} \text { whenever } U_{r} \geq 0 \text { for any } r \in[s, t) \\
Y_{t}-Y_{s} \text { whenever } U_{r}<0 \text { for any } r \in[s, t) .
\end{array}\right.
$$

They proved that the Laplace transform (11) for $Z=U$ takes the form

$$
\mathbb{E}_{x}^{U}\left(e^{-q \tau_{a}^{+}} ; \tau_{a}^{+}<\tau_{b}^{-}\right)=\frac{W_{U}^{(q)}(x, b)}{W_{U}^{(q)}(a, b)}
$$

where the function $W_{U}^{(q)}$ is defined by

$$
W_{U}^{(q)}(x, y)=W_{Y}^{(q)}(x-y)+\alpha 1_{(x \geq 0)} \int_{0}^{x} W_{X}^{(q)}(x-z) W_{Y}^{(q) \prime}(z-y) d z
$$

with $W_{Y}^{(q)}$ being the $q$-scale function of $Y$. They obtained, in addition, a representation of the potential measures of $U$ using scale functions of $X$ and $Y$.

A spectrally negative Lévy process can be regarded as the capital of an insurance company and applied to evaluate the risk of ruin. Hence it is sometimes called a Lévy insurance risk process. The Kyprianou-Loeffen's refracted Lévy process $U$ can be regarded as a modified insurance risk process when dividends are being paid out at a rate $\alpha$ during the period it exceeds 0 .

In this paper, we generalize Kyprianou-Loeffen's refracted Lévy processes. For two Lévy processes $X$ and $Y$ which may have different Lévy exponents, we construct a new refracted process whose positive and negative motions have the same law as $X$ and $Y$, respectively. More precisely,

$$
\left\{\begin{array}{l}
\text { If } x>0,\left(U_{t}\right)_{t \leq \tau_{0}^{-}} \text {under } \mathbb{P}_{x}^{U} \text { is equal in law to }\left(X_{t}\right)_{t \leq \tau_{0}^{-}} \text {under } \mathbb{P}_{x}^{X} \\
\text { If } x<0,\left(U_{t}\right)_{t \leq \tau_{0}^{+}} \text {under } \mathbb{P}_{x}^{U} \text { is equal in law to }\left(Y_{t}\right)_{t \leq \tau_{0}^{+}} \text {under } \mathbb{P}_{x}^{Y} .
\end{array}\right.
$$

One may expect that we can characterize the desired process as a solution to the following stochastic differential equation

$$
U_{t}-U_{0}=\int_{(0, t]} 1_{\left\{U_{s-} \geq 0\right\}} d X_{s}+\int_{(0, t]} 1_{\left\{U_{s-}<0\right\}} d Y_{s},
$$

where the driving noises $X$ and $Y$ are supposed to be independent. Although (1) for $Y_{t} \stackrel{d}{=} X_{t}+\alpha t$ is apparently different from (11) because of independence, their solutions are actually equivalent in law. When $X$ has bounded variation paths, we can construct a solution of (1) by a simple method of piecing excursions (see [10]); otherwise we do not know existence of a solution of (11). When $X$ and $Y$ are compound Poisson processes with positive drifts, uniqueness of the solution is easily proved because of the fact that the point 0 is irregular for itself for any solution $U$; otherwise we do not know uniqueness of a solution of (1). 
In this paper we utilize the excursion theory instead of a stochastic differential equation. Let $X$ and $Y$ be two spectrally negative Lévy processes. Suppose $X$ has unbounded variation paths and has no Gaussian component. We then define the excursion measure $n^{U}$ by

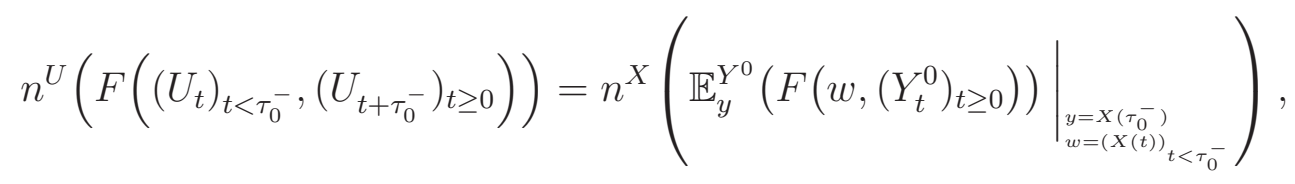

where $n^{X}$ stands for an excursion measure of $X$ and $Y_{t}^{0}=Y_{t \wedge T_{0}}$ for the stopped process of $Y$ upon hitting zero. We define the stopped process $\mathbb{P}_{x}^{U^{0}}$ by (1) with $n^{X}$ being replaced by $\mathbb{P}_{x}^{X}$. We can therefore construct a Feller process from $n^{U}$ together with the family of stopped processes $\left\{\mathbb{P}_{x}^{U^{0}}\right\}_{x \neq 0}$. As one of our main theorems, we show the Laplace transform (11) for the process $Z=U$, our new refracted Lévy process, takes the same form as (1) where $W_{U}^{(q)}$ will be defined in Theorem 6.2 in a more complicated form than (1). Note that $W_{U}^{(q)}$ 's will be represented using only Laplace exponents and scale functions of $X$ and $Y$. Furthermore, we will study the potential measures of $U$ with and without absorbing barriers.

We finally discuss approximation problem. Let $X$ and $Y$ be as in the previous paragraph. Let $X^{(n)}$ and $Y^{(n)}$ be the compound Poisson processes with positive drifts obtained from $X$ and $Y$, respectively, by removing small jumps of magnitude less than $\frac{1}{n}$. Assuming that $X^{(n)}$ and $Y^{(n)}$ are independent, we construct $U^{(n)}$ as the unique solution of (1). We thus show that $U^{(n)}$ converges to our refracted process $U$ in law on the space of càdlàg paths equipped with the Skorokhod topology.

The organization of the present paper is as follows. In Section 2 we propose some notation and recall preliminary facts about spectrally negative Lévy processes. In Section 3 we calculate several quantities related to excursion measures and scale functions. In Section 4 we recall Kyprianou-Loeffen's refracted Lévy processes. In Section 5 we define our new refracted Lévy processes. In Section 6 we study the exit problem of our refracted Lévy processes. In Section 7 we calculate the potential measures of our refracted Lévy processes killed upon exiting $[b, a]$. In Section 8 we study the approximation problem. In Section A we make a careful treatment of Markov property of our new process.

\section{Notation and preliminaries}

Let $\mathbb{D}$ denote the set of functions $\omega:[0, \infty) \rightarrow \mathbb{R}$ which are càdlàg. We equip $\mathbb{D}$ with the Skorokhod topology. Let $\mathcal{B}(\mathbb{D})$ denote the class of Borel sets of $\mathbb{D}$.

When we consider a process $Z=\left\{Z_{t}: t \geq 0\right\}=\{Z(t): t \geq 0\}$, we always write $\mathbb{P}_{x}^{Z}$ for the underlying probability measure for $Z$ starting from $x$. In addition to the passage times $\tau_{a}^{+}$and $\tau_{b}^{-}$defined in (1), we sometimes need the hitting time of a point $x \in \mathbb{R}$ denoted by

$$
T_{x}=\inf \left\{t>0: Z_{t}=x\right\}
$$


For $q>0, x \in \mathbb{R}$ and a non-negative or bounded measurable function $f$, we write

$$
R_{Z}^{(q)} f(x):=\mathbb{E}_{x}^{Z}\left(\int_{0}^{\infty} e^{-q t} f\left(Z_{t}\right) d t\right)
$$

We write $r_{Z}^{(q)}(x, y)$ for the resolvent density, if it exists, i.e.,

$$
R_{Z}^{(q)} f(x)=\int_{\mathbb{R}} r_{Z}^{(q)}(x, y) f(y) d y
$$

We sometimes settle a lower barrier $b<0$ and an upper barrier $a>0$. For $q>0, x \in \mathbb{R}$ and a non-negative or bounded measurable function $f$, we write

$$
\begin{array}{r}
\underline{\underline{R}}_{Z}^{(q ; b, a)} f(x):=\underline{\bar{R}}_{Z}^{(q)} f(x):=\mathbb{E}_{x}^{Z}\left(\int_{0}^{\tau_{a}^{+} \wedge \tau_{b}^{-}} e^{-q t} f\left(Z_{t}\right) d t\right), \\
\underline{R}_{Z}^{(q ; b)} f(x):=\underline{R}_{Z}^{(q)} f(x):=\mathbb{E}_{x}^{Z}\left(\int_{0}^{\tau_{b}^{-}} e^{-q t} f\left(Z_{t}\right) d t\right), \\
\bar{R}_{Z}^{(q ; a)} f(x):=\bar{R}_{Z}^{(q)} f(x):=\mathbb{E}_{x}^{Z}\left(\int_{0}^{\tau_{a}^{+}} e^{-q t} f\left(Z_{t}\right) d t\right) .
\end{array}
$$

We write $\underline{\underline{r}}_{Z}^{(q ; b, a)}(x, y)=\underline{\bar{r}}_{Z}^{(q)}(x, y), \underline{r}_{Z}^{(q ; b, a)}(x, y)=\underline{r}_{Z}^{(q)}(x, y)$ and $\bar{r}_{Z}^{(q ; b, a)}(x, y)=\bar{r}_{Z}^{(q)}(x, y)$ for the corresponding densities, if they exist.

Let $Z$ be a spectrally negative Lévy process, which is always assumed not to be monotone. Then it is well known that the Laplace exponent

$$
\Psi_{Z}(q):=\log \mathbb{E}_{0}^{Z}\left(e^{q Z_{1}}\right)
$$

is finite for all $q \geq 0$. We denote its right inverse by

$$
\Phi_{Z}(\theta)=\inf \left\{q \geq 0: \Psi_{Z}(q)=\theta\right\},
$$

which is finite for all $\theta \geq 0$. If $Z$ has bounded variation paths, the Laplace exponent is known to necessarily take the form

$$
\Psi_{Z}(q)=\delta_{Z} q-\int_{(-\infty, 0)}\left(1-e^{q y}\right) \Pi_{Z}(d y)
$$

for some constant $\delta_{Z}>0$ and some Lévy measure $\Pi_{Z}$ satisfying $\Pi_{Z}[0, \infty)=0$ and $\int_{(-\infty, 0)}(1 \wedge|y|) \Pi_{Z}(d y)<\infty$. If $Z$ has unbounded variation paths, the Laplace exponent is known to necessarily take the form

$$
\Psi_{Z}(q)=\gamma_{Z} q+\frac{\sigma_{Z}^{2}}{2} q^{2}-\int_{(-\infty, 0)}\left(1-e^{q y}+q y 1_{(-1,0)}(y)\right) \Pi_{Z}(d y)
$$

for some constants $\gamma_{Z} \in \mathbb{R}$ and $\sigma_{Z} \geq 0$ and some Lévy measure $\Pi_{Z}$ satisfying $\Pi_{Z}[0, \infty)=0$ and $\int_{(-\infty, 0)}\left(1 \wedge y^{2}\right) \Pi_{Z}(d y)<\infty$. 
Definition 2.1. For each $q \geq 0$, we define $W_{Z}^{(q)}: \mathbb{R} \rightarrow[0, \infty)$ such that $W_{Z}^{(q)}=0$ on $(-\infty, 0)$ and $W_{Z}^{(q)}$ on $[0, \infty)$ is continuous satisfying

$$
\int_{0}^{\infty} e^{-\beta x} W_{Z}^{(q)}(x) d x=\frac{1}{\Psi_{Z}(\beta)-q}
$$

for all $\beta>\Phi_{Z}(q)$. This function $W_{Z}^{(q)}$ is called the $q$-scale function of $Z$.

For the proof of unique existence and its basic facts listed below, see, e.g., [9]. For all $b<x<a$ and $q \geq 0$, we have

$$
\mathbb{E}_{x}^{Z}\left(e^{-q \tau_{a}^{+}} ; \tau_{a}^{+}<\tau_{b}^{-}\right)=\frac{W_{Z}^{(q)}(x-b)}{W_{Z}^{(q)}(a-b)}
$$

and

$$
\mathbb{E}_{x}^{Z}\left(e^{-q \tau_{a}^{+}} ; \tau_{a}^{+}<\infty\right)=e^{-\Phi_{Z}(q)(a-x)}
$$

It is known that, when $Z$ has bounded variation paths, we have

$$
W_{Z}^{(q)}(0)=\frac{1}{\delta_{Z}}
$$

for all $q \geq 0$. For all $q \geq 0$, we have

$$
\begin{gathered}
r_{Z}^{(q)}(x, y)=\Phi_{Z}^{\prime}(q) e^{-\Phi_{Z}(q)(y-x)}-W_{Z}^{(q)}(x-y), \quad x, y \in \mathbb{R}, \\
\underline{r}_{Z}^{(q ; b)}(x, y)=\underline{r}_{Z}^{(q)}(x, y)=e^{-\Phi_{Z}(q)(y-b)} W_{Z}^{(q)}(x-b)-W_{Z}^{(q)}(x-y), \quad x, y \in[b, \infty), \\
\bar{r}_{Z}^{(q ; a)}(x, y)=\bar{r}_{Z}^{(q)}(x, y)=e^{-\Phi_{Z}(q)(a-x)} W_{Z}^{(q)}(a-y)-W_{Z}^{(q)}(x-y), \quad x, y \in(-\infty, a]
\end{gathered}
$$

and

$$
\underline{\bar{r}}_{Z}^{(q ; b, a)}(x, y)=\underline{\bar{r}}_{Z}^{(q)}(x, y)=\frac{W_{Z}^{(q)}(x-b) W_{Z}^{(q)}(a-y)}{W_{Z}^{(q)}(a-b)}-W_{Z}^{(q)}(x-y), \quad x, y \in[b, a] .
$$

We write $\widetilde{\Pi}_{Z}$ for the measure carried on $(-\infty, 0) \times(0, \infty)$ defined by

$$
\widetilde{\Pi}_{Z}(d u d v):=1_{\{u<0, v>0\}} \Pi_{Z}(d u-v) d v .
$$

Theorem 2.2. (i) For all $0<x<\infty, q \geq 0$, and non-negative measurable function $f: \mathbb{R}^{2} \rightarrow[0, \infty)$, we have

$$
\mathbb{E}_{x}^{Z}\left(e^{-q \tau_{0}^{-}} f\left(Z_{\tau_{0}^{-}}, Z_{\tau_{0}^{-}-}\right) ; \tau_{0}^{-}<\infty, Z_{\tau_{0}^{-}}<0\right)=\int f(u, v) G_{Z}^{(q)}(x, d u d v),
$$


where $G_{Z}^{(q)}(x, \cdot)$ is the measure carried on $(-\infty, 0) \times(0, \infty)$ defined by

$$
G_{Z}^{(q)}(x, d u d v):=\underline{r}_{Z}^{(q ; 0)}(x, v) \widetilde{\Pi}_{Z}(d u d v) .
$$

(ii) For all $0<x<a, q \geq 0$, and non-negative measurable function $f$, we have

$$
\mathbb{E}_{x}^{Z}\left(e^{-q \tau_{0}^{-}} f\left(Z_{\tau_{0}^{-}}, Z_{\tau_{0}^{-}-}\right) ; \tau_{0}^{-}<\tau_{a}^{+}, Z_{\tau_{0}^{-}}<0\right)=\int f(u, v) \bar{G}_{Z}^{(q, a)}(x, d u d v),
$$

where $\bar{G}_{Z}^{(q, a)}(x, \cdot)$ is the measure carried on $(-\infty, 0) \times(0, \infty)$ defined by

$$
\bar{G}_{Z}^{(q, a)}(x, d u d v)=\bar{G}_{Z}^{(q)}(x, d u d v):=\underline{\bar{r}}_{Z}^{(q ; 0, a)}(x, v) \widetilde{\Pi}_{Z}(d u d v) .
$$

We omit the proof of Theorem 2.2 because it can be found in 9, Theorem 10.1 and Exercise 10.6] and also in [10, Theorem 5.5]. These kernels $G_{Z}^{(q)}$ and $\bar{G}_{Z}^{(q)}$ are called the Gerber-Shiu measures.

\section{Some calculations related to excursion measures and scale functions}

In this section, we make some calculations related to excursion measures and scale functions for a spectrally negative Lévy process $X$. See [11, [1] and [12 for recent studies on a close relation between $n^{X}$, i.e., the excursion measure of $X$ itself, and the excursion measure of the reflected of $X$.

We divide the discussion into the two cases of unbounded and of bounded variations.

(I) We assume that

$X$ has unbounded variation paths and have no Gaussian component.

Since 0 is regular for itself, $X$ has an excursion measure $n^{X}$ away from zero. We impose on $n^{X}$ the following normalization:

$$
n^{X}\left(1-e^{-q T_{0}}\right)=\frac{1}{r_{X}^{(q)}(0,0)}=\frac{1}{\Phi_{X}^{\prime}(q)}=\Psi_{X}^{\prime}\left(\Phi_{X}(q)\right) .
$$

Note that $n^{X}$ is carried on the set of càdlàg paths stopped upon hitting 0 . Note also that $n^{X}$ possesses the Markov property; for example,

$$
n^{X}\left(X_{s} \in B_{1}, X_{t} \in B_{2}\right)=n^{X}\left(1_{\left\{X_{s} \in B_{1}\right\}} \mathbb{P}_{X(s)}^{X^{0}}\left(X_{t-s}^{0} \in B_{2}\right)\right),
$$

for all $0<s<t$ and $B_{1}, B_{2} \in \mathcal{B}(\mathbb{R})$, where $X_{t}^{0}=X_{t \wedge T_{0}}$ denotes the stopped process of $X$ upon hitting zero. Since $X$ has no Gaussian component, we can see

$$
0<\tau_{0}^{-}<T_{0} \leq \infty \text { or } \tau_{0}^{-}=T_{0}=\infty \quad n^{X} \text {-a.e. }
$$

by [11, Theorem 3]. 
Theorem 3.1. For all $a>0$ and $q \geq 0$, we have

$$
n^{X}\left(e^{-q \tau_{a}^{+}} ; \tau_{a}^{+}<\infty\right)=\frac{1}{W_{X}^{(q)}(a)}
$$

In particular, we have

$$
n^{X}\left(\tau_{a}^{+}<\infty\right)=\frac{1}{W_{X}(a)},
$$

where $W_{X}:=W_{X}^{(0)}$.

Remark 3.2. The two ways of normalization (3) and (3.1) are natural analogies of those for diffusion processes. See [3, (2.5) and Theorem 3.1] and [16, (39) and Theorem 3.1].

Remark 3.3. The left-hand side of (3.1) may admit several other expressions, such as

$$
n^{X}\left(e^{-q \tau_{a}^{+}}\right)=n^{X}\left(e^{-q \tau_{a}^{+}} ; \tau_{a}^{+}<\infty\right)=n^{X}\left(e^{-q \tau_{a}^{+}} ; \tau_{a}^{+}<\tau_{0}^{-}\right) .
$$

The first equality of (3.3) follows from the fact that $e^{-q \tau_{a}^{+}}=0$ on $\left\{\tau_{a}^{+}=\infty\right\}$. Since $X$ has no positive jumps, the measure $n^{X}$ is supported on the disjoint union

$$
\left\{\tau_{a}^{+}<\tau_{0}^{-} \leq \infty\right\} \cup\left\{\tau_{0}^{-}<\tau_{a}^{+}=\infty\right\} \cup\left\{\tau_{0}^{-}=\tau_{a}^{+}=\infty\right\}
$$

Thus the sets $\left\{\tau_{a}^{+}<\infty\right\}$ and $\left\{\tau_{a}^{+}<\tau_{0}^{-}\right\}$are equal up to $n^{X}$-null sets, which yields the second equality of (3.3).

The following theorem can be regarded as the Gerber-Shiu measure for the excursion measure (see also [12]).

Theorem 3.4. For all $q \geq 0$ and non-negative measurable function $f$, we have

$$
n^{X}\left(e^{-q \tau_{0}^{-}} f\left(X_{\tau_{0}^{-}}, X_{\tau_{0}^{-}-}\right) ; \tau_{0}^{-}<\infty\right)=\int f(u, v) K_{X}^{(q)}(d u d v),
$$

where $K_{X}^{(q)}$ is the measure carried on $(-\infty, 0) \times(0, \infty)$ defined by

$$
K_{X}^{(q)}(d u d v)=e^{-\Phi_{X}(q) v} \widetilde{\Pi}_{X}(d u d v) .
$$

We prove Theorems 3.1 and 3.4 at the same time.

Proof of Theorems 3.1 and 3.4.

Step.1 We show that the quantity

$$
c:=n^{X}\left(e^{-q \tau_{a}^{+}} ; \tau_{a}^{+}<\infty\right) W_{X}^{(q)}(a)
$$


does not depend upon $a>0$ nor $q \geq 0$. First, we prove

$$
n^{X}\left(\tau_{a}^{+}<\infty\right) W_{X}(a)=n^{X}\left(e^{-q \tau_{a}^{+}} ; \tau_{a}^{+}<\infty\right) W_{X}^{(q)}(a)
$$

for all $a>0$ and $q \geq 0$. Using the monotone convergence theorem, we have

$$
\frac{n^{X}\left(e^{-q \tau_{a}^{+}} ; \tau_{a}^{+}<\infty\right)}{n^{X}\left(\tau_{a}^{+}<\infty\right)}=\lim _{\varepsilon \downarrow 0} \frac{n^{X}\left(e^{-q \tau_{\varepsilon}^{+}}\left(e^{-q \tau_{a}^{+}} 1_{\left\{\tau_{a}^{+}<\infty\right\}}\right) \circ \theta_{\tau_{\varepsilon}^{+}} ; \tau_{\varepsilon}^{+}<\infty\right)}{n^{X}\left(e^{-q \tau_{\varepsilon}^{+}}\left(1_{\left\{\tau_{a}^{+}<\infty\right\}}\right) \circ \theta_{\tau_{\varepsilon}^{+}} ; \tau_{\varepsilon}^{+}<\infty\right)} .
$$

Using the strong Markov property and (2), we have

$$
\text { (3) }=\lim _{\varepsilon \downarrow 0} \frac{n^{X}\left(e^{-q \tau_{\varepsilon}^{+}} ; \tau_{\varepsilon}^{+}<\infty\right) \mathbb{E}_{\varepsilon}^{X}\left(e^{-q \tau_{a}^{+}} 1_{\left\{\tau_{a}^{+}<\tau_{0}^{-}\right\}}\right)}{n^{X}\left(e^{-q \tau_{\varepsilon}^{+}} ; \tau_{\varepsilon}^{+}<\infty\right) \mathbb{P}_{\varepsilon}^{X}\left(\tau_{a}^{+}<\tau_{0}^{-}\right)}=\frac{W_{X}(a)}{W_{X}^{(q)}(a)},
$$

where we used $\lim _{\varepsilon \downarrow 0} W_{X}^{(q)}(\varepsilon) / W_{X}(\varepsilon)=1$ by [드, Lemma 1. (i)]. Second, we prove

$$
n^{X}\left(\tau_{a_{1}}^{+}<\infty\right) W_{X}\left(a_{1}\right)=n^{X}\left(\tau_{a_{2}}^{+}<\infty\right) W_{X}\left(a_{2}\right),
$$

for all $0<a_{1}<a_{2}$. This identity can be obtained by

$$
\frac{n^{X}\left(\tau_{a_{2}}^{+}<\infty\right)}{n^{X}\left(\tau_{a_{1}}^{+}<\infty\right)}=\frac{n^{X}\left(\left(\tau_{a_{2}}^{+}<\tau_{0}^{-}\right) \circ \theta_{\tau_{a_{1}}^{+}} ; \tau_{a_{1}}^{+}<\infty\right)}{n^{X}\left(\tau_{a_{1}}^{+}<\infty\right)}=\frac{W_{X}\left(a_{1}\right)}{W_{X}\left(a_{2}\right)}
$$

where we used the strong Markov property and (2).

Step.2 We show (3.4) with $K_{X}^{(q)}$ being multiplied by $c$. Using the monotone convergence theorem and the strong Markov property, we have

$$
\begin{aligned}
& n^{X}\left(e^{-q \tau_{0}^{-}} f\left(X_{\tau_{0}^{-}}, X_{\tau_{0}^{-}-}\right) ; \tau_{0}^{-}<\infty\right) \\
= & \lim _{\varepsilon \downarrow 0} n^{X}\left(e^{-q \tau_{\varepsilon}^{+}}\left(e^{-q \tau_{0}^{-}} f\left(X_{\tau_{0}^{-}}, X_{\tau_{0}^{-}-}\right) 1_{\left\{X_{\tau_{0}^{-}-}>\varepsilon\right\}}\right) \circ \theta_{\tau_{\varepsilon}^{+}} ; \tau_{\varepsilon}^{+}<\infty, \tau_{0}^{-}<\infty\right)
\end{aligned}
$$

and using $(i)$ of Theorem 2.2 and Step.1, we have

$$
\begin{aligned}
(3) & =\lim _{\varepsilon \downarrow 0} \frac{c}{W_{X}^{(q)}(\varepsilon)} W_{X}^{(q)}(\varepsilon) \int_{\varepsilon}^{\infty} d v \int_{(-\infty, 0)} e^{-\Phi_{X}(q) v} f(u, v) \Pi_{X}(d u-v) \\
& =\int f(u, v) c K_{X}^{(q)}(d u, d v) .
\end{aligned}
$$

Step.3 We show $c=1$. Since $X$ has no Gaussian component, i.e., $\sigma_{X}=0$, differentiating (2), we have

$$
\Psi_{X}^{\prime}(q)=\gamma_{X}+\int_{(-\infty, 0)}\left(y e^{q y}-y 1_{(-1,0)}(y)\right) \Pi_{X}(d y)
$$


for all $q>0$. Using (3), we have on one hand

$$
n^{X}\left(1-e^{-q T_{0}}\right)=\Psi_{X}^{\prime}\left(\Phi_{X}(q)\right)=\gamma_{X}+\int_{(-\infty, 0)}\left(y e^{\Phi_{X}(q) y}-y 1_{(-1,0)}(y)\right) \Pi_{X}(d y) .
$$

On the other hand, using the monotone convergence theorem and the strong Markov property, we have

$$
\begin{aligned}
& n^{X}\left(1-e^{-q T_{0}}\right) \\
= & n^{X}\left(\tau_{0}^{-}=\infty\right)+n^{X}\left(1-e^{-q T_{0}} ; \tau_{0}^{-}<\infty\right) \\
= & n^{X}\left(\tau_{1}^{+}<\infty\right) \lim _{p \uparrow \infty} \mathbb{E}_{1}^{X}\left(\tau_{p}^{+}<\tau_{0}^{-}\right)+n^{X}\left(1-e^{-q \tau_{0}^{-}} \mathbb{E}_{X\left(\tau_{0}^{-}\right)}^{X}\left(e^{-q T_{0}}\right) ; \tau_{0}^{-}<\infty\right) .
\end{aligned}
$$

Using (2), (2), Step.1 and Step.2, we have

$$
\begin{aligned}
(\underline{3}) & =n^{X}\left(\tau_{1}^{+}<\infty\right) \lim _{p \uparrow \infty} \frac{W_{X}(1)}{W_{X}(p)}+n^{X}\left(1-e^{-q \tau_{0}^{-}+\Phi_{X}(q) X\left(\tau_{0}^{-}\right)} ; \tau_{0}^{-}<\infty\right) \\
& =c \frac{1}{W_{X}(\infty)}+c \int\left(e^{-\Phi_{X}(0) v}-e^{\Phi_{X}(q)(u-v)}\right) \widetilde{\Pi}_{X}(d u d v) .
\end{aligned}
$$

Since it is known that

$$
W_{X}(\infty)= \begin{cases}\frac{1}{\Psi_{X}^{\prime}(0+)} & \mathbb{P}\left(\lim _{t \uparrow \infty} X_{t}=\infty\right)=1 \\ \infty & \text { otherwise }\end{cases}
$$

(see e.g., [9, pp.247]), we have

$$
\text { (3) }=c\left(\Psi_{X}^{\prime}(0+) \vee 0\right)+c \int_{(-\infty, 0)} \Pi_{X}(d u) \int_{0}^{-u}\left(e^{-\Phi_{X}(0) v}-e^{\Phi_{X}(q) u}\right) d v .
$$

We divide the remainder of the proof into two parts.

(i) Suppose $\Psi_{X}^{\prime}(0+)>0$. In this case, we have $\Phi_{X}(0)=0$ and so

$$
\text { (3) }=c \Psi_{X}^{\prime}(0+)+c \int_{(-\infty, 0)}\left(u e^{\Phi_{X}(q) u}-u\right) \Pi_{X}(d u) .
$$

Using (3), we have

$$
\text { (3) }=c\left(\gamma_{X}+\int_{(-\infty, 0)}\left(u e^{\Phi_{X}(q) u}-u 1_{(-1,0)}(u)\right) \Pi_{X}(d u)\right) .
$$

Using (3), we obtain $c=1$.

(ii) Suppose $\Psi_{X}^{\prime}(0+) \leq 0$. In this case, we have

$$
\text { (3) }=c \int_{(-\infty, 0)}\left(u e^{\Phi_{X}(q) u}+\frac{1}{\Phi_{X}(0)}-\frac{1}{\Phi_{X}(0)} e^{\Phi_{X}(0) u}\right) \Pi_{X}(d u) .
$$

Since $\Psi(\Phi(0))=0$ and by (2) with $\sigma_{X}=0$, we have

$$
(\underline{3})=c\left(\gamma_{X}+\int_{(-\infty, 0)}\left(u e^{\Phi_{X}(q) u}-u 1_{(-1,0)}(u)\right) \Pi_{X}(d u)\right) .
$$

Using (3), we obtain $c=1$. Thus the proof is complete. 
We need the following two lemmas for later use.

Lemma 3.5. For all $a>0, q \geq 0$ and non-negative measurable function $f$ we have

$$
n^{X}\left(e^{-q \tau_{0}^{-}} f\left(X_{\tau_{0}^{-}}, X_{\tau_{0}^{-}-}\right) ; \tau_{0}^{-}<\tau_{a}^{+}\right)=\int f(u, v) \bar{K}_{X}^{(q, a)}(d u d v),
$$

where $\bar{K}_{X}^{(q, a)}$ is the measure carried on $(-\infty, 0) \times(0, \infty)$ defined by

$$
\bar{K}_{X}^{(q, a)}(d u d v)=\bar{K}_{X}^{(q)}(d u d v):=\frac{W_{X}^{(q)}(a-v)}{W_{X}^{(q)}(a)} \widetilde{\Pi}_{X}(d u d v)
$$

The proof is parallel to that of $(i)$ of Lemma 2.2, so that we omit it.

Lemma 3.6. For all $q \geq 0$ and non-negative measurable function $f$, we have

$$
n^{X}\left(\int_{0}^{\tau_{0}^{-} \wedge T_{0}} e^{-q t} f\left(X_{t}\right) d t\right)=\int_{0}^{\infty} e^{-\Phi_{X}(q) y} f(y) d y
$$

Proof. Using the monotone convergence theorem, we have

$$
n^{X}\left(\int_{0}^{\tau_{0}^{-} \wedge T_{0}} e^{-q t} f\left(X_{t}\right) d t\right)=\lim _{\varepsilon \downarrow 0} n^{X}\left(\int_{\tau_{\varepsilon}^{+}}^{\tau_{0}^{-} \wedge T_{0}} e^{-q t} f\left(X_{t}\right) d t ; \tau_{\varepsilon}^{+}<\infty\right)
$$

and using the strong Markov property, we have

$$
\begin{aligned}
(\underline{3}) & =\lim _{\varepsilon \downarrow 0} n^{X}\left(e^{-q \tau_{\varepsilon}^{+}}\left(\int_{0}^{\tau_{0}^{-} \wedge T_{0}} e^{-q t} f\left(X_{t}\right) d t\right) \circ \theta_{\tau_{\varepsilon}^{+}} ; \tau_{\varepsilon}^{+}<\infty\right) \\
& =\lim _{\varepsilon \downarrow 0} n^{X}\left(e^{-q \tau_{\varepsilon}^{+}} ; \tau_{\varepsilon}^{+}<\infty\right) \mathbb{E}_{\varepsilon}^{X}\left(\int_{0}^{\tau_{0}^{-} \wedge T_{0}} e^{-q t} f\left(X_{t}\right) d t\right) \\
& =\lim _{\varepsilon \downarrow 0} \frac{1}{W_{X}^{(q)}(\varepsilon)} \mathbb{E}_{\varepsilon}^{X}\left(\int_{0}^{\tau_{0}^{-} \wedge T_{0}} e^{-q t} f\left(X_{t}\right) d t\right),
\end{aligned}
$$

where in (3) we used Theorem 3.1, Using (2) with $b=0$, we obtain

$$
\int_{\varepsilon}^{\infty} f(y) e^{-\Phi_{X}(q) y} d y \leq \frac{1}{W_{X}^{(q)}(\varepsilon)} \mathbb{E}_{\varepsilon}^{X}\left(\int_{0}^{\tau_{0}^{-}} e^{-q t} f\left(X_{t}\right) d t\right) \leq \int_{0}^{\infty} f(y) e^{-\Phi_{X}(q) y} d y .
$$

By the monotone convergence theorem, the proof is complete.

(II) We assume that $X$ has bounded variation paths. Note that in this case 0 is irregular for itself. We write

$$
n^{X}=\delta_{X} \mathbb{P}_{0}^{X^{0}}
$$


Then we have

$$
n^{X}\left(e^{-q \tau_{a}^{+}} ; \tau_{a}^{+}<\infty\right)=\delta_{X} \mathbb{E}_{0}^{X^{0}}\left(e^{-q \tau_{a}^{+}} ; \tau_{a}^{+}<\infty\right)=\delta_{X} \frac{W_{X}^{(q)}(0)}{W_{X}^{(q)}(a)}=\frac{1}{W_{X}^{(q)}(a)},
$$

where we used (2) and (2). Thus we see that Theorem 3.1 still holds in this case. Lemmas 3.4, 3.5 and 3.6 still hold as they are by a similar argument. In particular, we obtain

$$
n^{X}\left(1-e^{-q T_{0}}\right)=\delta_{X} \mathbb{E}_{0}^{X^{0}}\left(1-e^{-q T_{0}}\right)=\frac{1}{\Phi_{X}^{\prime}(q)}=\Psi_{X}^{\prime}\left(\Phi_{X}(q)\right) .
$$

which may be regarded as the counterpart of the normalization (3) in the unbounded case.

\section{Kyprianou-Loeffen's Refracted Lévy processes}

Let us recall some results of Kyprianou-Loeffen [10]. We fix a constant $\alpha>0$ and let $X$ be a general spectrally negative Lévy process, which may possibly have Gaussian component. Set $Y_{t}=X_{t}+\alpha t$. Note that $0<\delta_{X}<\delta_{X}+\alpha=\delta_{Y}$ if $X$ has bounded variation paths.

Theorem 4.1 ([10]). For a fixed starting point $U_{0}=x \in \mathbb{R}$, there exists a unique strong solution to (11) $D$

Let $U$ be a solution to Kyprianou-Loeffen's stochastic differential equation (11).

Theorem $4.2([10])$. For all $x \in[b, a]$ and $q \geq 0$, we have (11) where $W_{U}^{(q)}$ is defined by (11).

They also calculated the potential densities with and without barriers.

Theorem $4.3([10])$. For all $x \in[b, a], q>0$, we have

$$
\begin{gathered}
\underline{\bar{r}}_{U}^{(q)}(x, y)= \begin{cases}\frac{W_{U}^{(q)}(x, b)}{W_{U}^{(q)}(a, b)} W_{X}^{(q)}(a-y)-W_{X}^{(q)}(x-y) & y \in(0, a] \\
\frac{W_{U}^{(q)}(x, b)}{W_{U}^{(q)}(a, b)} W_{U}^{(q)}(a, y)-W_{U}^{(q)}(x, y) & y \in[b, 0],\end{cases} \\
\underline{r}_{U}^{(q)}(x, y)= \begin{cases}\frac{W_{U}^{(q)}(x, b)}{\alpha \frac{H_{U}^{(q)}(b)}{(q)}} e^{-\Phi_{X}(q) y}-W_{X}^{(q)}(x-y) & y \in(0, \infty) \\
\frac{\underline{H}_{U}^{(q)}(y)}{\underline{H}_{U}^{(q)}(b)} W_{U}^{(q)}(x, b)-W_{U}^{(q)}(x, y) & y \in[b, 0]\end{cases}
\end{gathered}
$$

with $\underline{H}_{U}^{(q)}(y)=\int_{0}^{\infty} e^{-\Phi_{X}(q) z} W_{Y}^{(q) \prime}(z-y) d z$,

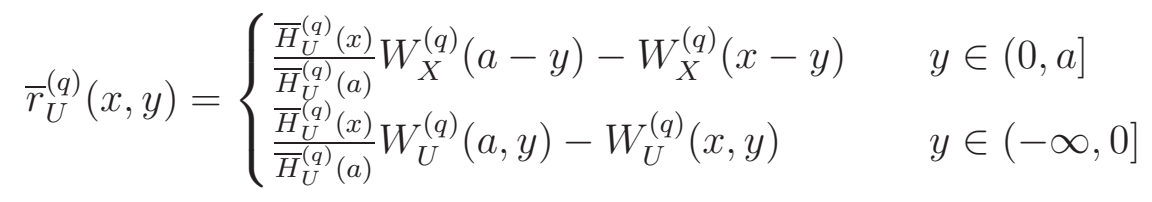


with $\bar{H}_{U}^{(q)}(x)=e^{\Phi_{Y}(q) x}+\alpha \Phi_{Y}(q) \int_{0}^{x} e^{\Phi_{Y}(q) z} W_{X}^{(q)}(x-z) d z$, and

$$
r_{U}^{(q)}(x, y)= \begin{cases}\frac{1}{\alpha} H_{U}^{(q)}(x) e^{-\Phi_{X}(q) y}-W_{X}^{(q)}(x-y) & y \in(0, \infty) \\ H_{U}^{(q)}(x) \underline{H}_{U}^{(q)}(y)-W_{U}^{(q)}(x, y) & y \in(-\infty, 0]\end{cases}
$$

with $H_{U}^{(q)}(x)=\bar{H}_{U}^{(q)}(x) \frac{\Phi_{X}(q)-\Phi_{Y}(q)}{\Phi_{Y}(q)}$, where $W_{U}^{(q)}$ has been given in (11).

\section{Generalization of refracted Lévy processes}

We now generalize Kyprianou-Loeffen's refracted Lévy processes. We assume that $X$ and $Y$ are spectrally negative Lévy processes. We assume, in addition, that

$X$ has no Gaussian component whenever $X$ has unbounded variation paths.

In the unbounded variation case, we define the law of the stopped process $\mathbb{P}_{x}^{U^{0}}$ by

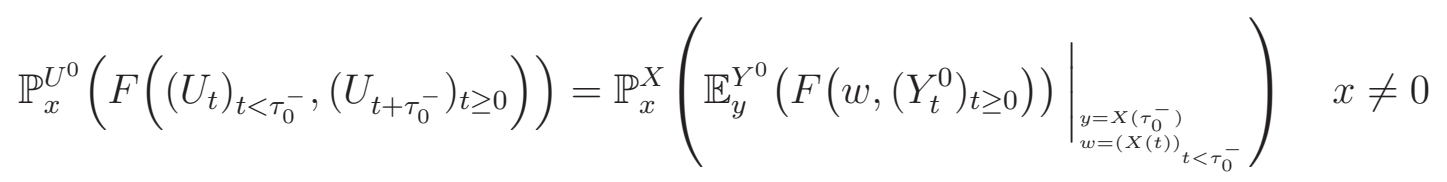

and the excursion measure $n^{U}$ by

$$
n^{U}\left(F\left(\left(U_{t}\right)_{t<\tau_{0}^{-}},\left(U_{t+\tau_{0}^{-}}\right)_{t \geq 0}\right)\right)=n^{X}\left(\left.\mathbb{E}_{y}^{Y^{0}}\left(F\left(w,\left(Y_{t}^{0}\right)_{t \geq 0}\right)\right)\right|_{\substack{y=X\left(\tau_{0}^{-}\right) \\ w=(X(t)) \\ t<\tau_{0}^{-}}}\right)
$$

for all non-negative measurable functional $F$, where $Y_{t}^{0}=Y_{t \wedge T_{0}}$ denotes the stopped process of $Y$ upon hitting zero. Thus, we appeal to the excursion theory (see Section $\mathrm{A}$ ), to construct the strong Markov process $U$ without stagnancy at 0 (that is, $R_{U}^{(1)} 1_{\{0\}}=0$ ) from $n^{U}$ together with $\left\{\mathbb{P}_{x}^{U^{0}}\right\}_{x \neq 0}$.

In the bounded variation case, we define $U$ as a solution of (1) constructed connecting $X$ and $Y$ mutually (this argument is similar as [10]). When $X$ and $Y$ are compound Poisson processes, uniqueness of the solution of (1) is easily proved. We write

$$
n^{X}=\delta_{X} \mathbb{P}_{0}^{X^{0}} \text { and } n^{U}=\delta_{X} \mathbb{P}_{0}^{U^{0}} .
$$

Then we obtain (11) as a formula. Therefore we can do a simultaneous discussion in between the two cases of bounded and of unbounded variation.

Theorem 5.1. For all $q>0$ and non-negative measurable function $f$ with $f(0)=0$, we have

$$
\begin{aligned}
N_{U}^{(q)} f & :=n^{U}\left(\int_{0}^{T_{0}} e^{-q t} f\left(X_{t}\right) d t\right) \\
& =\int_{0}^{\infty} e^{-\Phi_{X}(q) y} f(y) d y+\int R_{Y^{0}}^{(q)} f(u) K_{X}^{(q)}(d u d v) .
\end{aligned}
$$


Consequently we have

$$
\begin{aligned}
R_{U}^{(q)} f(0) & =\frac{N_{U}^{(q)} f}{q N_{U}^{(q)} 1}, \\
R_{U}^{(q)} f(x) & =R_{Y^{0}}^{(q)} f(x)+e^{\Phi_{Y}(q) x} R_{U}^{(q)} f(0), \quad x<0,
\end{aligned}
$$

and

$$
R_{U}^{(q)} f(x)=\underline{R}_{X}^{(q ; 0)} f(x)+\int R_{U}^{(q)} f(u) G_{X}^{(q)}(x, d u d v), \quad x>0,
$$

where

$$
\underline{R}_{X}^{(q ; 0)} f(x)=\mathbb{E}_{x}^{X}\left(\int_{0}^{\tau_{0}^{-}} e^{-q t} f\left(X_{t}\right) d t\right)
$$

Proof. Let us calculate $N_{U}^{(q)} f$. Since $\int_{0}^{T_{0}}=\int_{0}^{\tau_{0}^{-}}+\int_{\tau_{0}^{-}}^{T_{0}}$, we have that $N_{U}^{(q)} f$ is equal to

$$
n^{U}\left(\int_{0}^{\tau_{0}^{-}} e^{-q t} f\left(U_{t}\right) d t\right)+n^{U}\left(e^{-q \tau_{0}^{-}}\left(\int_{0}^{T_{0}} e^{-q t} f\left(U_{t}\right) d t\right) \circ \theta_{\tau_{0}^{-}} ; \tau_{0}^{-}<\infty\right) .
$$

Using Lemmas 3.6 and 3.4, we obtain (5.1).

Let us prove (5.1). Note that the finiteness of $N_{U}^{(q)} 1$ will be proved in Lemma A.4. When $X$ has unbounded variation paths, the formula (5.1) can be found, e.g., in [14, pp.423]. Suppose $X$ has bounded variation paths. We denote $T_{0}^{(0)}=0$ and define

$$
T_{0}^{(n)}=\inf \left\{t>T_{0}^{(n-1)}: X_{t}=0\right\}
$$

recursively for all $n \in \mathbb{N}$. Then we have

$$
\begin{aligned}
R_{U}^{(q)} f(0) & =\sum_{n=0}^{\infty} \mathbb{E}_{0}^{U}\left(\int_{T_{0}^{(n)}}^{T_{0}^{(n+1)}} e^{-q t} f\left(U_{t}\right) d t ; T_{0}^{(n)}<\infty\right) \\
& =\sum_{n=0}^{\infty} \mathbb{E}_{0}^{U}\left(e^{-q T_{0}}\right)^{n} \mathbb{E}_{0}^{U}\left(\int_{0}^{T_{0}} e^{-q t} f\left(U_{t}\right) d t\right) \\
& =\frac{\mathbb{E}_{0}^{U^{0}}\left(\int_{0}^{T_{0}} e^{-q t} f\left(U_{t}\right) d t\right)}{q \mathbb{E}_{0}^{U^{0}}\left(\int_{0}^{T_{0}} e^{-q t} d t\right)} .
\end{aligned}
$$

Since we write $n^{U}=\delta_{X} \mathbb{P}_{0}^{U^{0}}$, we obtain (5.1).

The remainder of the proof is straightforward.

The following theorem shows the choice of $n^{U}$ leads to a normalization similar to (3)). 
Theorem 5.2. For all $q>0$, we have

$$
\begin{aligned}
n^{U}\left(1-e^{-q T_{0}}\right) & =\frac{1}{r_{U}^{(q)}(0,0+)} \\
& =\left(\Psi_{X}^{\prime}(0) \vee 0\right)+\int\left(e^{\Phi_{X}(0) u}-e^{\Phi_{Y}(q) u-\Phi_{X}(q) v}\right) \widetilde{\Pi}_{X}(d u d v) .
\end{aligned}
$$

Proof. By (5.1) of Theorem 5.1, we have

$$
r_{U}^{(q)}(0, y)=\frac{1}{q N_{U}^{(q)} 1}\left(e^{-\Phi_{X}(q) y} 1_{(y>0)}+\int r_{Y^{0}}^{(q)}(u, y) K_{X}^{(q)}(d u d v)\right) .
$$

Since $r_{Y^{0}}^{(q)}(u, y)=0$ for $u<0$ and $y>0$, we have

$$
r_{U}^{(q)}(0,0+)=\frac{1}{q N_{U}^{(q)} 1}
$$

On the other hand, we have

$$
q N_{U}^{(q)} 1=n^{U}\left(1-e^{-q T_{0}}\right)
$$

by the definition of $N_{U}^{(q)}$. Thus we obtain (5.2).

The other expression (5.2) can be proved easily by a similar argument to (3).

\section{Exit Problem of generalized refracted Lévy processes}

We prepare a general formula.

Lemma 6.1. Let $Z$ be a standard process with no positive jumps without stagnancy at 0 (i.e., $R_{Z}^{(1)} 1_{\{0\}}=0$ ). If 0 is regular for itself, then

$$
\mathbb{E}_{0}^{Z}\left(e^{-q \tau_{a}^{+}} ; \tau_{a}^{+}<\tau_{b}^{-}\right)=\frac{n^{Z}\left(e^{-q \tau_{a}^{+}} ; \tau_{a}^{+}<\infty\right)}{n^{Z}\left(1-e^{-q T_{0}} 1_{\left\{\tau_{a}^{+}=\infty, \tau_{b}^{-}=\infty\right\}}\right)}
$$

for all $a>0>b$ and $q \geq 0$, where $n^{Z}$ denotes an excursion measure away from 0 . If 0 is irregular for itself, the identity (6.1) still holds where $n^{Z}$ denotes a constant multiple of $\mathbb{P}_{0}^{Z^{0}}$.

Proof. It is sufficient to prove (6.1) only when $q>0$. We assume first that 0 is regular for itself. Let $p$ denote a Poisson point process defined on the probability space $(\Omega, \mathcal{F}, \mathbb{P})$ with characteristic measure $n^{Z}$. Set $\eta(s)=\sum_{u \leq s} T_{0}(p(u))$. Note that $\eta$ will be the inverse local time at 0 for the process constructed from the excursions, which equals in law to $Z$ under $\mathbb{P}_{0}^{Z}$. For $E \in \mathcal{B}(\mathbb{D})$, we write $\kappa_{E}=\inf \{s \geq 0: p(s) \in E\}$. We let 
$A=\left\{\tau_{a}^{+}<\infty\right\} \cup\left\{\tau_{b}^{-}<\infty\right\}$ and we denote by $\varepsilon^{*}=p\left(\kappa_{A}\right)$ the first excursion belonging to $A$. Then we have

$$
\begin{aligned}
\mathbb{E}_{0}^{Z}\left(e^{-q \tau_{a}^{+}} ; \tau_{a}^{+}<\tau_{b}^{-}\right) & =\mathbb{E}\left(e^{-q \eta\left(\kappa_{A}-\right)} e^{-q \tau_{a}^{+}\left(\varepsilon^{*}\right)}\right) \\
& =\mathbb{E}\left(e^{-q \eta\left(\kappa_{A}-\right)}\right) \frac{n^{Z}\left(e^{-q \tau_{a}^{+}} ; A\right)}{n^{Z}(A)} \\
& =\mathbb{E}\left(e^{-q \eta\left(\kappa_{A}-\right)}\right) \frac{n^{Z}\left(e^{-q \tau_{a}^{+}} ; \tau_{a}^{+}<\infty\right)}{n^{Z}(A)}
\end{aligned}
$$

where $\mathbb{E}$ denotes the expectation with respect to $\mathbb{P}$. Note that in (6) we used the renewal property of the Poisson point process and in (6) we used the fact that $e^{-q \tau_{a}^{+}}=0$ on $\left\{\tau_{a}^{+}=\infty, \tau_{b}^{-}<\infty\right\}$. We write $p_{A^{c}}$ for $p$ restricted to excursions belonging to $A^{c}$ and write $\eta_{A^{c}}(s)=\sum_{u \leq s} T_{0}\left(p_{A^{c}}(u)\right)$. Since $\eta\left(\kappa_{A}-\right)=\eta_{A^{c}}\left(\kappa_{A}\right)$ where $\eta_{A^{c}}$ and $\kappa_{A}$ are independent, we have

$$
\begin{aligned}
\mathbb{E}\left(e^{-q \eta\left(\kappa_{A}-\right)}\right) & =n^{Z}(A) \int_{0}^{\infty} e^{-n^{Z}(A) t} \mathbb{E}\left(e^{-q \eta_{A^{c}}(t)}\right) d t \\
& =n^{Z}(A) \int_{0}^{\infty} e^{-n^{Z}(A) t}\left(\exp \left(-t n^{Z}\left(1-e^{-q T_{0}} ; A^{c}\right)\right)\right) d t \\
& =\frac{n^{Z}(A)}{n^{Z}\left(1-e^{-q T_{0}} 1_{A^{c}}\right)} .
\end{aligned}
$$

Thus we obtain (6.1).

We second assume that 0 is irregular for itself. Using the notation of the proof of Theorem 5.1, we have

$$
\begin{aligned}
\mathbb{E}_{0}^{Z}\left(e^{-q \tau_{a}^{+}} ; \tau_{a}^{+}<\tau_{b}^{-}\right) & =\sum_{n=0}^{\infty} \mathbb{E}_{0}^{Z}\left(e^{-q \tau_{a}^{+}} ; T_{0}^{(n)}<\tau_{a}^{+}<\left(T_{0}^{(n+1)} \wedge \tau_{b}^{-}\right)\right) \\
& =\sum_{n=0}^{\infty} \mathbb{E}_{0}^{Z^{0}}\left(e^{-q T_{0}} ; \tau_{a}^{+}=\infty, \tau_{b}^{-}=\infty\right)^{n} \mathbb{E}_{0}^{Z^{0}}\left(e^{-q \tau_{a}^{+}} ; \tau_{a}^{+}<\infty\right) \\
& =\frac{\mathbb{E}_{0}^{Z^{0}}\left(e^{-q \tau_{a}^{+}} ; \tau_{a}^{+}<\infty\right)}{1-\mathbb{E}_{0}^{Z 0}\left(e^{-q T_{0}} ; \tau_{a}^{+}=\infty, \tau_{b}^{-}=\infty\right)}
\end{aligned}
$$

Thus we obtain (6.1).

Theorem 6.2. For all $x \in[b, a]$ and $q \geq 0$, we have

$$
\mathbb{E}_{x}^{U}\left(e^{-q \tau_{a}^{+}} ; \tau_{a}^{+}<\tau_{b}^{-}\right)=\frac{W_{U}^{(q)}(x, b)}{W_{U}^{(q)}(a, b)},
$$

where the function $W_{U}^{(q)}(x, y)$ is defined as follows: for $x \in(0, \infty)$,

$$
\begin{aligned}
W_{U}^{(q)}(x, y)= & W_{X}^{(q)}(x) W_{Y}^{(q)}(-y)\left(\Psi_{X}^{\prime}(0) \vee 0\right) \\
& +\int\left(W_{X}^{(q)}(x) W_{Y}^{(q)}(-y) e^{\Phi_{X}(0) u}-W_{Y}^{(q)}(u-y) W_{X}^{(q)}(x-v)\right) \widetilde{\Pi}_{X}(d u d v)
\end{aligned}
$$


and for $x \in(-\infty, 0]$,

$$
W_{U}^{(q)}(x, y)=W_{Y}^{(q)}(x-y)
$$

Proof. We discuss the two cases of unbounded and of bounded variation at the same time.

We calculate $\mathbb{E}_{0}^{U}\left(e^{-q \tau_{a}^{+}} ; \tau_{a}^{+}<\tau_{b}^{-}\right)$. Using Lemma 6.1, we have

$$
\mathbb{E}_{0}^{U}\left(e^{-q \tau_{a}^{+}} ; \tau_{a}^{+}<\tau_{b}^{-}\right)=\frac{n^{U}\left(e^{-q \tau_{a}^{+}} ; \tau_{a}^{+}<\infty\right)}{n^{U}\left(1-e^{-q T_{0}} 1_{\left\{\tau_{a}^{+}=\infty, \tau_{b}^{-}=\infty\right\}}\right)} .
$$

Using Theorem 3.1, we can rewrite the numerator as

$$
n^{U}\left(e^{-q \tau_{a}^{+}} ; \tau_{a}^{+}<\infty\right)=n^{X}\left(e^{-q \tau_{a}^{+}} ; \tau_{a}^{+}<\infty\right)=\frac{1}{W_{X}^{(q)}(a)} .
$$

We divide the denominator $n^{U}\left(1-e^{-q T_{0}} 1_{\left\{\tau_{a}^{+}=\infty, \tau_{b}^{-}=\infty\right\}}\right)$ into the following sum:

$$
n^{U}\left(1-e^{-q T_{0}}\right)+n^{U}\left(e^{-q T_{0}} ;\left\{\tau_{a}^{+}<\infty\right\} \cap\left\{\tau_{b}^{-}=\infty\right\}\right)+n^{U}\left(e^{-q T_{0}} ; \tau_{b}^{-}<\infty\right) .
$$

Let us compute these expectations. For the second term, we have

$$
\begin{aligned}
& n^{U}\left(e^{-q T_{0}} ;\left\{\tau_{a}^{+}<\infty\right\} \cap\left\{\tau_{b}^{-}=\infty\right\}\right) \\
= & n^{U}\left(e^{-q \tau_{a}^{+}}\left(e^{-q T_{0}} 1_{\left\{\tau_{b}^{-}=\infty\right\}}\right) \circ \theta_{\tau_{a}^{+}} ; \tau_{a}^{+}<\infty\right) \\
= & n^{X}\left(e^{-q \tau_{a}^{+}} ; \tau_{a}^{+}<\infty\right) \mathbb{E}_{a}^{X}\left(e^{-q \tau_{0}^{-}} \mathbb{E}_{X\left(\tau_{0}^{-}\right)}^{Y}\left(e^{-q T_{0}} 1_{\left\{T_{0}<\tau_{b}^{-}\right\}}\right) ; \tau_{0}^{-}<\infty\right) \\
= & \frac{1}{W_{X}^{(q)}(a)} \int \frac{W_{Y}^{(q)}(u-b)}{W_{Y}^{(q)}(-b)} G_{X}^{(q)}(a, d u d v),
\end{aligned}
$$

where in (6) we used Theorem 3.1, (i) of Theorem 2.2 and (2)). For the third term, we have

$$
n^{U}\left(e^{-q T_{0}} ; \tau_{b}^{-}<\infty\right)=n^{U}\left(e^{-q \tau_{0}^{-}} \mathbb{E}_{U\left(\tau_{0}^{-}\right)}^{U}\left(e^{-q T_{0}} ; \tau_{b}^{-}<T_{0}\right) ; \tau_{0}^{-}<\infty\right) .
$$

Using Lemma 3.4, we have

$$
\begin{aligned}
(6) & =\int e^{-\Phi_{X}(q) v} \mathbb{E}_{u}^{Y}\left(e^{-q T_{0}} ; \tau_{b}^{-}<T_{0}\right) \widetilde{\Pi}_{X}(d u d v) \\
& =\int e^{-\Phi_{X}(q) v}\left(e^{\Phi_{Y}(q) u}-\frac{W_{Y}^{(q)}(u-b)}{W_{Y}^{(q)}(-b)}\right) \widetilde{\Pi}_{X}(d u d v),
\end{aligned}
$$

where in (6) we used (2) and (21). Therefore, using (5.2), we obtain

$$
n^{U}\left(1-e^{-q T_{0}} 1_{\left\{\tau_{a}^{+}=\infty, \tau_{b}^{-}=\infty\right\}}\right)=\frac{1}{W_{X}^{(q)}(a)} \frac{W_{U}^{(q)}(a, b)}{W_{U}^{(q)}(0, b)}
$$


and we obtain (6.2) for $x=0$. For all $x<0$, we have

$$
\mathbb{E}_{x}^{U}\left(e^{-q \tau_{a}^{+}} ; \tau_{a}^{+}<\tau_{b}^{-}\right)=\mathbb{E}_{x}^{Y}\left(e^{-q \tau_{0}^{+}} ; \tau_{0}^{+}<\tau_{b}^{-}\right) \mathbb{E}_{0}^{U}\left(e^{-q \tau_{a}^{+}} ; \tau_{a}^{+}<\tau_{b}^{-}\right) .
$$

Using (21) and (6.2) for $x=0$, we have (6.2) for $x<0$. For all $x>0$, we have

$$
\begin{aligned}
& \mathbb{E}_{x}^{U}\left(e^{-q \tau_{a}^{+}} ; \tau_{a}^{+}<\tau_{b}^{-}\right) \\
= & \mathbb{E}_{x}^{U}\left(e^{-q \tau_{a}^{+}} ; \tau_{a}^{+}<\tau_{0}^{-}\right)+\mathbb{E}_{x}^{U}\left(e^{-q \tau_{0}^{-}}\left(e^{-q \tau_{a}^{+}} 1_{\left\{\tau_{a}^{+}<\tau_{b}^{-}\right\}}\right) \circ \theta_{\tau_{0}^{-}} ; \tau_{0}^{-}<\tau_{a}^{+}\right) \\
= & \frac{W_{X}^{(q)}(x)}{W_{X}^{(q)}(a)}+\mathbb{E}_{x}^{X}\left(e^{-q \tau_{0}^{-}} \mathbb{E}_{X\left(\tau_{0}^{-}\right)}^{Y}\left(e^{-q \tau_{a}^{+}} ; \tau_{a}^{+}<\tau_{b}^{-}\right) ; \tau_{0}^{-}<\tau_{a}^{+}\right),
\end{aligned}
$$

where in (6) we used (2). Using (6.2) for $x<0$ and (ii) of Theorem 2.2, the second term of (6) is equal to

$$
\int \frac{W_{U}^{(q)}(u, b)}{W_{U}^{(q)}(a, b)} \underline{\underline{x}}_{X}^{(q ; 0, a)}(x, v) \widetilde{\Pi}_{X}(d u d v) .
$$

Thus we obtain (6.2) for $x>0$. The proof is complete.

Corollary 6.3. For all $x \in(-\infty, a]$ and $q \geq 0$, we have

$$
\mathbb{E}_{x}^{U}\left(e^{-q \tau_{a}^{+}}\right)=\frac{\bar{W}_{U}^{(q)}(x)}{\bar{W}_{U}^{(q)}(a)}
$$

where the function $\bar{W}_{U}^{(q)}(x)$ is defined as follows: for $x \in(0, \infty)$,

$$
\begin{aligned}
\bar{W}_{U}^{(q)}(x)= & W_{X}^{(q)}(x)\left(\Psi_{X}^{\prime}(0) \vee 0\right) \\
& +\int\left(W_{X}^{(q)}(x) e^{\Phi_{X}(0) u}-W_{X}^{(q)}(x-v) e^{\Phi_{Y}(q) u}\right) \widetilde{\Pi}_{X}(d u d v)
\end{aligned}
$$

and for $x \in(-\infty, 0]$,

$$
\bar{W}_{U}^{(q)}(x)=e^{\Phi_{Y}(q) x} .
$$

In particular, $\bar{W}_{U}^{(q)}(x)$ is a continuous and increasing function of $x$.

Proof. Using the monotone convergence theorem and Theorem 6.2, we have

$$
\mathbb{E}_{x}^{U}\left(e^{-q \tau_{a}^{+}}\right)=\lim _{b \downarrow-\infty} \mathbb{E}_{x}^{U}\left(e^{-q \tau_{a}^{+}} ; \tau_{a}^{+}<\tau_{b}^{-}\right)=\lim _{b \downarrow-\infty} \frac{W_{U}^{(q)}(x, b) / W_{Y}^{(q)}(-b)}{W_{U}^{(q)}(a, b) / W_{Y}^{(q)}(-b)}
$$

Using the last equality of [7, pp.124], we have

$$
\lim _{b \downarrow-\infty}\left(W_{U}^{(q)}(x, b) / W_{Y}^{(q)}(-b)\right)=\bar{W}_{U}^{(q)}(x),
$$


and we have (6.3) .

Next, we prove that $\bar{W}_{U}^{(q)}$ is increasing and continuous. It is obvious that $\bar{W}_{U}^{(q)}$ is increasing and continuous on $(-\infty, 0]$, since $\bar{W}_{U}^{(q)}(x)=e^{\Phi_{Y}(q) x}$. Using the dominated convergence theorem, we have

$$
\lim _{\varepsilon \downarrow 0} \bar{W}_{U}^{(q)}(\varepsilon)=\lim _{\varepsilon \downarrow 0} \frac{1}{\mathbb{E}_{0}^{U}\left(e^{-q \tau_{\varepsilon}^{+}}\right)}=\frac{1}{\mathbb{E}_{0}^{U}\left(\lim _{\varepsilon \downarrow 0} e^{-q \tau_{\varepsilon}^{+}}\right)}=1
$$

so that we see $\bar{W}_{U}^{(q)}$ is continuous at 0 . Since

$$
\bar{W}_{U}^{(q)}(x)=\frac{1}{\mathbb{E}_{0}^{U}\left(e^{-q \tau_{x}^{+}}\right)},
$$

it is thus sufficient to prove that $\mathbb{E}_{0}^{U}\left(e^{-q \tau_{x}^{+}}\right)$is decreasing and continuous on $(0, \infty)$. For $0<x<y$, we have

$$
\mathbb{E}_{0}^{U}\left(e^{-q \tau_{x}^{+}}\right)-\mathbb{E}_{0}^{U}\left(e^{-q \tau_{y}^{+}}\right)=\mathbb{E}_{0}^{U}\left(e^{-q \tau_{x}^{+}}\right)\left(1-\mathbb{E}_{x}^{U}\left(e^{-q \tau_{y}^{+}}\right)\right) \geq 0 .
$$

Using (2), for $x>0$, we have

$$
\begin{aligned}
\limsup _{\varepsilon \downarrow 0}\left|\mathbb{E}_{0}^{U}\left(e^{-q \tau_{x-\varepsilon}^{+}}\right)-\mathbb{E}_{0}^{U}\left(e^{-q \tau_{x+\varepsilon}^{+}}\right)\right| & =\limsup _{\varepsilon \downarrow 0} \mathbb{E}_{0}^{U}\left(e^{-q \tau_{x-\varepsilon}^{+}}\right)\left(1-\mathbb{E}_{x-\varepsilon}^{U}\left(e^{-q \tau_{x+\varepsilon}^{+}}\right)\right) \\
& \leq \limsup _{\varepsilon \downarrow 0}\left(1-\mathbb{E}_{x-\varepsilon}^{X}\left(e^{-q \tau_{x+\varepsilon}^{+}} ; \tau_{x+\varepsilon}^{+}<\tau_{0}^{-}\right)\right) \\
& =\left(1-\lim _{\varepsilon \downarrow 0} \frac{W_{X}^{(q)}(x-\varepsilon)}{W_{X}^{(q)}(x+\varepsilon)}\right)=0 .
\end{aligned}
$$

The proof is complete.

Let $C_{0}$ denote the set of continuous functions $f: \mathbb{R} \rightarrow \mathbb{R}$ which vanish at $+\infty$ and $-\infty$. Note that $C_{0}$ is a Banach space with respect to the supremum norm $\|f\|=\sup _{x \in \mathbb{R}}|f(x)|$ for $f \in C_{0}$.

Theorem 6.4. Our generalized refracted Lévy process is a Feller process.

Proof. Since $R_{U}^{(q)}$ comes from transition operators, it is sufficient to verify the following conditions:

(i) For all $q>0, R_{U}^{(q)}$ is a map from $C_{0}$ to $C_{0}$.

(ii) For all $f \in C_{0}, \lim _{q \uparrow \infty}\left\|q R_{U}^{(q)} f-f\right\|=0$.

1) The proof of $(i)$ 
First, we prove that $R_{U}^{(q)} f$ is continuous. Let $x \in \mathbb{R}$ and $\varepsilon>0$. Noting that $U$ has no positive jump, we have

$$
\begin{aligned}
& \left|R_{U}^{(q)} f(x+\varepsilon)-R_{U}^{(q)} f(x)\right| \\
& \leq\left|R_{U}^{(q)} f(x+\varepsilon)-\mathbb{E}_{x}^{U}\left(e^{-q \tau_{x+\varepsilon}^{+}}\right) R_{U}^{(q)} f(x+\varepsilon)\right|+\left|\mathbb{E}_{x}^{U}\left(\int_{0}^{\tau_{x+\varepsilon}^{+}} e^{-q t} f\left(U_{t}\right) d t\right)\right| \\
& \leq\left|R_{U}^{(q)} f(x+\varepsilon)\right|\left(1-\mathbb{E}_{x}^{U}\left(e^{-q \tau_{x+\varepsilon}^{+}}\right)\right)+\|f\| \mathbb{E}_{x}^{U}\left(\int_{0}^{\tau_{x+\varepsilon}^{+}} e^{-q t} d t\right) \\
& \leq \frac{2}{q}\|f\|\left(1-\mathbb{E}_{x}^{U}\left(e^{-q \tau_{x+\varepsilon}^{+}}\right)\right) .
\end{aligned}
$$

By Corollary 6.3, we have

$$
\text { (6) })=\frac{2}{q}\|f\|\left(1-\frac{\bar{W}_{U}^{(q)}(x)}{\bar{W}_{U}^{(q)}(x+\varepsilon)}\right) \rightarrow 0 \text { as } \varepsilon \downarrow 0 \text {. }
$$

Thus we obtain right-continuity of $R_{U}^{(q)} f$. For the left-continuity we have

$$
\begin{aligned}
& \left|R_{U}^{(q)} f(x-\varepsilon)-R_{U}^{(q)} f(x)\right| \\
& \leq\left|\mathbb{E}_{x-\varepsilon}^{U}\left(e^{-q \tau_{x}^{+}}\right) R_{U}^{(q)} f(x)-R_{U}^{(q)} f(x)\right|+\left|\mathbb{E}_{x-\varepsilon}^{U}\left(\int_{0}^{\tau_{x}^{+}} e^{-q t} f\left(U_{t}\right) d t\right)\right|
\end{aligned}
$$

and the remainder of its proof is similar to that of the right-continuity.

Second, we prove that $R_{U}^{(q)} f$ vanishes at $-\infty$. For $x<0$, we may rewrite (5.1) as

$$
R_{U}^{(q)} f(x)=R_{Y}^{(q)} f(x)-e^{\Phi_{Y}(q) x} R_{Y}^{(q)} f(0)+e^{\Phi_{Y}(q) x} R_{U}^{(q)} f(0) .
$$

By the Feller property of $Y$, we see that $\lim _{x \downarrow-\infty} R_{U}^{(q)} f(x)=0$.

Third, we prove that $R_{U}^{(q)} f$ vanishes at $+\infty$. We may assume without loss of generality that $f \geq 0$. For all $x>0$, we have

$$
\begin{aligned}
R_{U}^{(q)} f(x) & =\mathbb{E}_{x}^{U}\left(\left(\int_{0}^{\tau_{0}^{-}}+\int_{\tau_{0}^{-}}^{\infty}\right) e^{-q t} f\left(U_{t}\right) d t\right) \\
& \leq R_{X}^{(q)} f(x)+\mathbb{E}_{x}^{X}\left(e^{-q \tau_{0}^{-}} R_{U}^{(q)} f\left(X_{\tau_{0}^{-}}\right)\right) \cdot \frac{1}{q}\|f\| .
\end{aligned}
$$

By the Feller property of $X$ and by the fact that $\mathbb{E}_{x}^{X}\left(e^{-q \tau_{0}^{-}}\right)=\mathbb{E}_{0}^{X}\left(e^{-q \tau_{-x}^{-}}\right) \rightarrow 0$ as $x \rightarrow \infty$, we obtain $\lim _{x \uparrow \infty} R_{U}^{(q)} f(x)=0$.

2) The proof of $(i i)$ 
Define

$$
\omega_{\varepsilon}(f ; x)=\sup _{y:|y-x| \leq \varepsilon}|f(y)-f(x)|
$$

Let us prove the pointwise convergence:

$$
\lim _{q \uparrow \infty} q R_{U}^{(q)} f(x)=f(x), \quad x \in \mathbb{R} .
$$

For $x \in \mathbb{R}$ and $\varepsilon>0$, we have

$$
\begin{aligned}
& \left|q R_{U}^{(q)} f(x)-f(x)\right| \\
& \leq q \mathbb{E}_{x}^{U}\left(\left(\int_{0}^{\tau_{x+\varepsilon}^{+} \wedge \tau_{x-\varepsilon}^{-}}+\int_{\tau_{x+\varepsilon}^{+} \wedge \tau_{x-\varepsilon}^{-}}^{\infty}\right) e^{-q t}\left|f\left(U_{t}\right)-f(x)\right| d t\right) \\
& \leq \mathbb{E}_{x}^{U}\left(1-e^{-q\left(\tau_{x+\varepsilon}^{+} \wedge \tau_{x-\varepsilon}^{-}\right)}\right) \omega_{\varepsilon}(f ; x)+2\|f\| \mathbb{E}_{x}^{U}\left(e^{-q\left(\tau_{x+\varepsilon}^{+} \wedge \tau_{x-\varepsilon}^{-}\right)}\right) .
\end{aligned}
$$

We thus obtain $\lim \sup _{q \uparrow \infty}\left|q R_{U}^{(q)} f(x)-f(x)\right| \leq \omega_{\varepsilon}(f ; x)$ for all $\varepsilon>0$, which proves ([6). By a standard argument with the help of the fact that the dual space of $C_{0}$ can be identified with the space of signed measures, we can see that $R_{U}^{(p)}\left(C_{0}\right)$ is dense in $C_{0}$ for all $p>0$.

Let $f=R_{U}^{(1)} g$ for some $g \in C_{0}$. Using the resolvent equation, we have

$$
\left\|f-q R_{U}^{(q)} f\right\|=\left\|R_{U}^{(q)} g-R_{U}^{(q)} f\right\| \leq \frac{1}{q}\|g-f\| \rightarrow 0, \text { as } q \uparrow \infty .
$$

Since $R_{U}^{(1)}\left(C_{0}\right)$ is dense in $C_{0}$, we obtain claim $(i i)$.

The proof is now complete.

\section{Potential measure of killed refracted Lévy processes}

In this section, we calculate the potential measure of refracted Lévy processes killed on exiting $[b, a]$.

Theorem 7.1. For all $x \in[b, a]$ and $q \geq 0$, we have

$$
\underline{\bar{x}}_{U}^{(q)}(x, y)= \begin{cases}\frac{W_{U}^{(q)}(x, b)}{W_{U}^{(q)}(a, b)} W_{X}^{(q)}(a-y)-W_{X}^{(q)}(x-y), & y \in(0, a] \\ \frac{W_{U}^{(q)}(x, b)}{W_{U}^{(q)}(a, b)} W_{U}^{(q)}(a, y)-W_{U}^{(q)}(x, y), & y \in[b, 0) .\end{cases}
$$

Proof. We follow the notation of Lemma 6.1 for $L, \eta$, $\kappa$, etc. 
Step.1 We calculate in the case $x=0$. When $X$ has unbounded variation paths, we have

$$
\begin{aligned}
& \mathbb{E}_{0}^{U}\left(\int_{0}^{\tau_{a}^{+} \wedge \tau_{b}^{-}} e^{-q t} f\left(U_{t}\right) d t\right) \\
= & \mathbb{E}_{0}^{U}\left(\int_{(0, \infty)} e^{-q s} 1_{\left\{s<\eta\left(\kappa_{A}-\right)\right\}} d L(s)\right) n^{U}\left(\int_{0}^{T_{0} \wedge \tau_{a}^{+} \wedge \tau_{b}^{-}} e^{-q t} f\left(U_{t}\right) d t\right) .
\end{aligned}
$$

where we used the compensation theorem of the excursion point process. We may rewrite (17) using $\eta_{A^{c}}$, as

$$
\mathbb{E}_{0}^{U}\left(\int_{0}^{\infty} e^{-q \eta_{A^{c}}(t)} 1_{\left\{t<\kappa_{A}\right\}} d t\right) n^{U}\left(\int_{0}^{T_{0} \wedge \tau_{a}^{+} \wedge \tau_{b}^{-}} e^{-q t} f\left(U_{t}\right) d t\right),
$$

the first factor of which equals to

$$
\int_{0}^{\infty} e^{-t n^{U}\left(1-e^{-q T_{0}} ; A^{c}\right)} e^{-t n^{U}(A)} d t=\frac{1}{n^{U}\left(1-e^{-q T_{0}} 1_{\left\{A^{c}\right\}}\right)} .
$$

When $X$ has bounded variation paths, we have

$$
\begin{aligned}
& \mathbb{E}_{0}^{U}\left(\int_{0}^{\tau_{a}^{+} \wedge \tau_{b}^{-}} e^{-q t} f\left(U_{t}\right) d t\right) \\
= & \sum_{n=0}^{\infty} \mathbb{E}_{0}^{U}\left(\int_{T_{0}^{(n)}}^{T_{0}^{(n+1)} \wedge \tau_{a}^{+} \wedge \tau_{b}^{-}} e^{-q t} f\left(U_{t}\right) d t ; T_{0}^{(n)}<\tau_{a}^{+} \wedge \tau_{b}^{-}\right) \\
= & \sum_{n=0}^{\infty} \mathbb{E}_{0}^{U}\left(e^{-q T_{0}} ; \tau_{a}^{+}=\infty, \tau_{b}^{-}=\infty\right)^{n} \mathbb{E}_{0}^{U}\left(\int_{0}^{T_{0} \wedge \tau_{a}^{+} \wedge \tau_{b}^{-}} e^{-q t} f\left(U_{t}\right) d t\right) \\
= & \frac{\mathbb{E}_{0}^{U^{0}}\left(\int_{0}^{T_{0} \wedge \tau_{a}^{+} \wedge \tau_{b}^{-}} e^{-q t} f\left(U_{t}\right) d t\right)}{1-\mathbb{E}_{0}^{U^{0}}\left(e^{-q T_{0}} ; \tau_{a}^{+}=\infty, \tau_{b}^{-}=\infty\right)},
\end{aligned}
$$

where we used the notation of the proof of Theorem [5.1. Since $n^{U}=\delta_{X} \mathbb{E}_{0}^{U}$, we obtain

$$
\mathbb{E}_{0}^{U}\left(\int_{0}^{\tau_{a}^{+} \wedge \tau_{b}^{-}} e^{-q t} f\left(U_{t}\right) d t\right)=\frac{n^{U}\left(\int_{0}^{T_{0} \wedge \tau_{a}^{+} \wedge \tau_{b}^{-}} e^{-q t} f\left(U_{t}\right) d t\right)}{n^{U}\left(1-e^{-q T_{0}} ; \tau_{a}^{+}<\infty, \tau_{b}^{-}=\infty\right)},
$$

which has the same form as in the case of unbounded variation. The denominator has already computed in ([6). Let us compute the numerator. In the case $f=1_{\left(a^{\prime}, a\right]}$ for $0<a^{\prime}<a$, we have

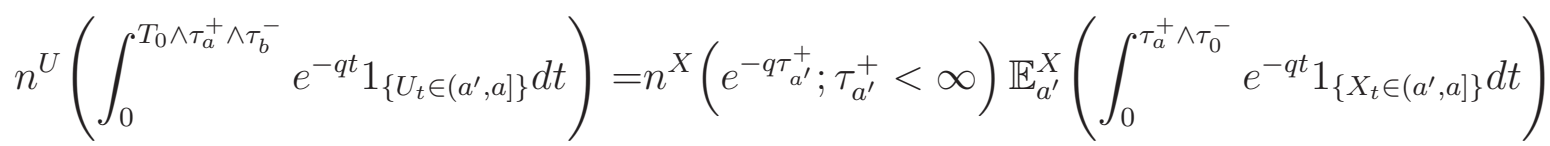

$$
\begin{aligned}
& =\frac{1}{W_{X}^{(q)}(a)} \int_{\left(a^{\prime}, a\right]} W_{X}^{(q)}(a-y) d y
\end{aligned}
$$


where in (7) we used Theorem 3.1 and (2). Thus we obtain (17.1) for $x=0$ and $y \in(0, a]$. In the case $f=1_{\left[b, b^{\prime}\right)}$ for $b<b^{\prime}<0$, we have

$n^{U}\left(\int_{0}^{T_{0} \wedge \tau_{a}^{+} \wedge \tau_{b}^{-}} e^{-q t} 1_{\left\{U_{t} \in\left[b, b^{\prime}\right)\right\}} d t\right)=n^{X}\left(e^{-q \tau_{0}^{-}} \mathbb{E}_{X\left(\tau_{0}^{-}\right)}^{Y}\left(\int_{0}^{\tau_{b}^{-} \wedge T_{0}} e^{-q t} 1_{\left\{Y_{t} \in\left[b, b^{\prime}\right)\right\}} d t\right) ; \tau_{0}^{-}<\tau_{a}^{+}\right)$.

Using Lemma 3.5, we have

$$
\text { (17) }=\int_{b}^{b^{\prime}}\left(\int \underline{\bar{r}}_{Y}^{(q ; b, 0)}(u, y) \frac{W_{X}^{(q)}(a-v)}{W_{X}^{(q)}(a)} \widetilde{\Pi}_{X}(d u d v)\right) d y \text {. }
$$

Using (6), (77) and (7), we obtain (17.1) for $x=0$ and $y \in[b, 0)$.

Step. 2 We calculate in the case $x<0$. We have

$$
\begin{aligned}
& \mathbb{E}_{x}^{U}\left(\int_{0}^{\tau_{a}^{+} \wedge \tau_{b}^{-}} e^{-q t} f\left(U_{t}\right) d t\right) \\
= & \mathbb{E}_{x}^{Y}\left(\int_{0}^{\tau_{0}^{+} \wedge \tau_{b}^{-}} e^{-q t} f\left(Y_{t}\right) d t\right)+\mathbb{E}_{x}^{U}\left(e^{-q \tau_{0}^{+}}\left(\int_{0}^{\tau_{a}^{+} \wedge \tau_{b}^{-}} e^{-q t} f\left(U_{t}\right) d t\right) \circ \theta_{\tau_{0}^{+}} ; \tau_{0}^{+}<\tau_{b}^{-}\right) .
\end{aligned}
$$

Using (2), we have that the first term equals to

$$
\int_{b}^{0} f(y) \underline{\underline{r}}_{Y}^{(q ; b, 0)}(x, y) d y
$$

Using (2) and Step.1, we have that the second term equals to

$$
\begin{aligned}
& \mathbb{E}_{x}^{Y}\left(e^{-q \tau_{0}^{+}} ; \tau_{0}^{+}<\tau_{b}^{-}\right) \mathbb{E}_{0}^{U}\left(\int_{0}^{\tau_{a}^{+} \wedge \tau_{b}^{-}} e^{-q t} f\left(U_{t}\right) d t\right) \\
= & \frac{W_{U}^{(q)}(x, b)}{W_{U}^{(q)}(a, b)}\left(\int_{0}^{a} f(y) W_{X}^{(q)}(a-y) d y\right. \\
& \left.+\int_{b}^{0} f(y)\left(\int W_{X}^{(q)}(a-v) \times \underline{\underline{r}}_{Y}^{(q ; b, 0)}(u, y) \widetilde{\Pi}_{X}(d u d v)\right) d y .\right)
\end{aligned}
$$

Using (7) and (7), we obtain (7.1) for $x<0$.

Step.3 We calculate in the case $x>0$. We have

$$
\begin{aligned}
& \mathbb{E}_{x}^{U}\left(\int_{0}^{\tau_{a}^{+} \wedge \tau_{b}^{-}} e^{-q t} f\left(U_{t}\right) d t\right) \\
= & \mathbb{E}_{x}^{X}\left(\int_{0}^{\tau_{0}^{-} \wedge \tau_{a}^{+}} e^{-q t} f\left(X_{t}\right) d t\right)+\mathbb{E}_{x}^{U}\left(e^{-q \tau_{0}^{-}}\left(\int_{0}^{\tau_{a}^{+} \wedge \tau_{b}^{-}} e^{-q t} f\left(U_{t}\right) d t\right) \circ \theta_{\tau_{0}^{-}} ; \tau_{0}^{-}<\tau_{a}^{+}\right) .
\end{aligned}
$$


Using (21), we have the first term equals to

$$
\int_{0}^{a} f(y) \underline{\underline{x}}_{X}^{(q ; 0, a)}(x, y) d y
$$

The second term equals to

$$
\begin{aligned}
& \mathbb{E}_{x}^{X}\left(e^{-q \tau_{0}^{-}} \mathbb{E}_{X\left(\tau_{0}^{-}\right)}^{U}\left(\int_{0}^{\tau_{a}^{+} \wedge \tau_{b}^{-}} e^{-q t} f\left(U_{t}\right) d t\right) ; \tau_{0}^{-}<\tau_{a}^{+}\right) \\
= & \int \mathbb{E}_{u}^{U}\left(\int_{0}^{\tau_{a}^{+} \wedge \tau_{b}^{-}} e^{-q t} f\left(U_{t}\right) d t\right) \underline{\underline{r}}_{X}^{(q ; 0, a)}(x, v) \widetilde{\Pi}_{X}(d u d v),
\end{aligned}
$$

where in (7) we used $(i i)$ of Theorem 2.2 . If $f$ is 0 on $(-\infty, 0]$, we have

$$
\text { (7) }=\left(\frac{W_{U}^{(q)}(x, b)}{W_{U}^{(q)}(a, b)}-\frac{W_{X}^{(q)}(x)}{W_{X}^{(q)}(a)}\right) \int_{0}^{\infty} f(y) W_{X}^{(q)}(a-y) d y \text {. }
$$

From (7) and (7) we obtain (7.1) for $x>0$ and $y \in(0, a]$. If $f$ is 0 on $(0, \infty)$, we have

$$
\text { (17) }=\int_{0}^{\infty} f(y)\left(\frac{W_{U}^{(q)}(x, b)}{W_{U}^{(q)}(a, b)} W_{U}^{(q)}(a, y)-W_{U}^{(q)}(x, y)\right) d y \text {. }
$$

Thus we obtain (7.1) for $x>0$ and $y \in[b, 0)$.

\section{Approximation problem}

Let $Z$ be a spectrally negative Lévy process. Let $\Psi_{Z}$ denote the Laplace exponent represented by (2). For $n \in \mathbb{N}$, we define

$$
\begin{aligned}
\Psi_{Z^{(n)}}(q)=\gamma_{Z} q & -\sigma_{Z^{2}}^{2} n^{2}\left(1-e^{q\left(-\frac{1}{n}\right)}+q\left(-\frac{1}{n}\right)\right) \\
& -\int_{\left(-\infty,-\frac{1}{n}\right)}\left(1-e^{q y}+q y 1_{\left(-1,-\frac{1}{n}\right)}(y)\right) \Pi_{Z}(d y) \\
= & \delta_{Z^{(n)}} q-\int_{(-\infty, 0)}\left(1-e^{q y}\right) \Pi_{Z^{(n)}}(d y)
\end{aligned}
$$

where

$$
\begin{aligned}
& \delta_{Z^{(n)}}=\gamma_{Z}+\sigma_{Z}^{2} n+\int_{\left(-1,-\frac{1}{n}\right)}(-y) \Pi_{Z}(d y) \\
& \Pi_{Z^{(n)}}=1_{\left(-\infty,-\frac{1}{n}\right)} \Pi_{Z}+\sigma_{Z}^{2} n^{2} \delta_{\left(-\frac{1}{n}\right)} .
\end{aligned}
$$

If we denote by $Z^{(n)}$ a Lévy process with Laplace exponent $\Psi_{Z^{(n)}}$, it is actually a compound Poisson process with positive drift. We note that $\Psi_{Z^{(n)}}(q) \rightarrow \Psi_{Z}(q)$ for all $q \geq 0$, so that 
we have $Z^{(n)} \rightarrow Z$ in law on $\mathbb{D}$. More precisely, by Bertoin [2, pp.210], we see that there exists a coupling of $Z^{(n)}$ 's such that $Z^{(n)} \rightarrow Z$ uniformly on compact intervals almost surely, which we will call the uniformly convergent coupling.

Let $X$ and $Y$ be spectrally negative Lévy processes and suppose that $X$ has unbounded variation paths and no Gaussian component. For each $n \in \mathbb{N}$, let $X^{(n)}$ and $Y^{(n)}$ be independent Lévy processes with Laplace exponents $\Psi_{X^{(n)}}$ and $\Psi_{Y^{(n)}}$, respectively. Let $U^{(n)}$ be defined as a unique strong solution of the stochastic differential equation

$$
U_{t}^{(n)}=U_{0}^{(n)}+\int_{(0, t]} 1_{\left\{U_{s-}^{(n)} \geq 0\right\}} d X_{s}^{(n)}+\int_{(0, t]} 1_{\left\{U_{s-}^{(n)}<0\right\}} d Y_{s}^{(n)}
$$

Theorem 8.1. $\left(U^{(n)}, \mathbb{P}_{x}^{U^{(n)}}\right)$ converges in distribution to $\left(U, \mathbb{P}_{x}^{U}\right)$ for all $x \in \mathbb{R}$.

We postpone the proof of Theorem 8.1 until the proof of Theorem 8.5 ,

Remark 8.2. We may expect

$$
\delta_{X^{(n)}} \mathbb{P}^{X^{(n) 0}} \rightarrow n^{X} \text { and } \delta_{X^{(n)}} \mathbb{P}^{U^{(n) 0}} \rightarrow n^{U} .
$$

The precise statements are as follows: For all bounded continuous function $f$, we have

$$
\delta_{X^{(n)}} \mathbb{E}_{0}^{X^{(n) 0}}\left(\int_{0}^{T_{0}} e^{-q t} f\left(X_{t}^{(n)}\right) d t\right) \rightarrow n^{X}\left(\int_{0}^{T_{0}} e^{-q t} f\left(X_{t}\right) d t\right) \text { as } n \uparrow \infty
$$

and

$$
\delta_{X^{(n)}} \mathbb{E}_{0}^{U^{(n) 0}}\left(\int_{0}^{T_{0}} e^{-q t} f\left(U_{t}^{(n)}\right) d t\right) \rightarrow n^{U}\left(\int_{0}^{T_{0}} e^{-q t} f\left(U_{t}\right) d t\right) \text { as } n \uparrow \infty .
$$

The proofs of these formulas are straightforward, so we omit it.

Lemma 8.3. For all non-positive $x^{(n)}$ and $x$ satisfying $x^{(n)} \rightarrow x$ as $n \uparrow \infty$ and for all $q>0$ and bounded continuous function $f$, we have

$$
R_{Y^{(n) 0}}^{(q)} f\left(x^{(n)}\right) \rightarrow R_{Y^{0}}^{(q)} f(x) \text { as } n \uparrow \infty .
$$

Proof. Using the strong Markov property, we have

$$
R_{Y^{(n) 0}}^{(q)} f\left(x^{(n)}\right)=R_{Y^{(n)}}^{(q)} f\left(x^{(n)}\right)-\mathbb{E}_{x^{(n)}}^{Y^{(n)}}\left(e^{-q \tau_{0}^{+}}\right) R_{Y^{(n)}}^{(q)} f(0)
$$

and a similar identity for $R_{Y^{0}}^{(q)} f(x)$. Using the uniformly convergent coupling and the dominated convergence theorem, we have $R_{Y^{(n)}}^{(q)} f\left(x^{(n)}\right)=R_{x^{(n)}+Y^{(n)}}^{(q)} f(0) \rightarrow R_{x+Y}^{(q)} f(0)=$ $R_{Y}^{(q)} f(x)$. Since $\Psi_{Y^{(n)}} \rightarrow \Psi_{Y}$ pointwise as $n \uparrow \infty$, we have $\Phi_{Y^{(n)}} \rightarrow \Phi_{Y}$ pointwise as $n \uparrow \infty$ and thus

$$
\mathbb{E}_{x^{(n)}}^{Y^{(n)}}\left(e^{-q \tau_{0}^{+}}\right)=e^{-\Phi_{Y}(n)}(q) x^{(n)} \rightarrow e^{-\Phi_{Y}(q) x}=\mathbb{E}_{x}^{Y}\left(e^{-q \tau_{0}^{+}}\right) .
$$

Thus we obtain (8.3). 
Theorem 8.4. For all $x \in \mathbb{R}, q>0$ and bounded continuous function $f$, we have

$$
R_{U^{(n)}}^{(q)} f(x) \rightarrow R_{U}^{(q)} f(x) \text { as } n \uparrow \infty .
$$

Proof. We may assume without loss of generality that $0 \leq f \leq 1$. We write $\rho_{X}:=$ $\inf _{n \in \mathbb{N}} \Phi_{X^{(n)}}(q)$ and $\rho_{Y}:=\inf _{n \in \mathbb{N}} \Phi_{Y^{(n)}}(q)$. Since $\Phi_{Z}(q)$ is strictly positive for all spectrally negative Lévy process $Z$, we have $\rho_{X}$ and $\rho_{Y}$ are strictly positive.

We prove (8.4) for $x=0$. By (5.1) and (5.1) of Theorem 5.1, it is sufficient to prove

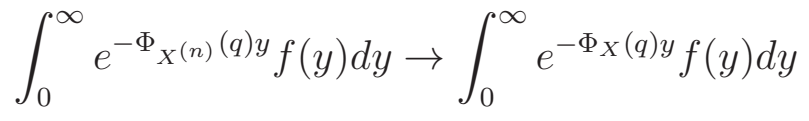

and

$$
\int R_{Y^{(n) 0}}^{(q)} f(u) K_{X^{(n)}}^{(q)}(d u d v) \rightarrow \int R_{Y^{0}}^{(q)} f(u) K_{X}^{(q)}(d u d v)
$$

Using $\Phi_{X^{(n)}} \rightarrow \Phi_{X}$ and the dominated convergence theorem, we have (8). Let us prove (8). Using (3.4) with $c=1$ and changing variables, we have

$$
\begin{aligned}
& \int R_{Y^{(n) 0}}^{(q)} f(u) K_{X^{(n)}}^{(q)}(d u d v) \\
= & \int_{(-\infty, 0)} \Pi_{X}(d u) 1_{\left(u<-\frac{1}{n}\right)} \int_{0}^{-u} e^{-\Phi_{X^{(n)}}(q) v} R_{Y^{(n) 0}}^{(q)} f(u+v) d v
\end{aligned}
$$

and a similar identity for $\left(Y^{0}, X\right)$. We have

$$
\begin{aligned}
& \left|1_{\left(u<-\frac{1}{n}\right)} \int_{0}^{-u} e^{-\Phi_{X^{(n)}}(q) v} R_{Y^{(n) 0}}^{(q)} f(u+v) d v\right| \\
\leq & \int_{0}^{-u} e^{-\rho_{X} v} \mathbb{E}_{u+v}^{Y^{(n)}}\left(\int_{0}^{T_{0}} e^{-q t} d t\right) d v \\
\leq & \frac{1}{q} \int_{0}^{-u} e^{-\rho_{X} v}\left(1-e^{\Phi_{Y^{(n)}}(q)(u+v)}\right) d v \\
\leq & \frac{1}{q} \int_{0}^{-u} e^{-\rho_{X} v}\left(1-e^{\rho_{Y}(u+v)}\right) d v \\
\leq & \frac{1}{q \rho_{X}}\left(1-e^{\rho_{X} u}\right)\left(1-e^{\rho_{Y} u}\right) \in L^{1}\left(\Pi_{X}\right) .
\end{aligned}
$$

Thus we may apply the dominated convergence theorem to obtain

$$
\lim _{n \uparrow \infty}(\sqrt[8]{6)})=\int_{(-\infty, 0)} \Pi_{X}(d u) \int_{0}^{-u} e^{-\Phi_{X}(q) v}\left(R_{Y^{0}}^{(q)} f(u+v)\right) d v,
$$

which shows (8). Thus we obtain (8.4) for $x=0$.

For $x<0$, (8.4) is obvious by (5.1) of Theorem 5.1 and Lemma 8.3. 
We prove (8.4) for $x>0$. By (5.1) of Theorem [5.1, it suffices to prove

$$
\underline{R}_{X(n)}^{(q ; 0)} f(x) \rightarrow \underline{R}_{X}^{(q ; 0)} f(x) \quad \text { as } n \uparrow \infty
$$

and

$$
\mathbb{E}_{x}^{X^{(n)}}\left(e^{-q \tau_{0}^{-}} R_{U^{(n)}}^{(q)} f\left(X_{\tau_{0}^{-}}^{(n)}\right)\right) \rightarrow \mathbb{E}_{x}^{X}\left(e^{-q \tau_{0}^{-}} R_{U}^{(q)} f\left(X_{\tau_{0}^{-}}\right)\right) \text {as } n \uparrow \infty .
$$

Note that $e^{-q \tau_{0}^{-}}=e^{-q \tau_{0}^{-}} 1_{\left(\tau_{0}^{-}<\infty\right)}$ a.s. Since $X$ has no Gaussian component, we have

$$
\inf _{t \in\left[0, \tau_{0}^{-}(X)\right)} X_{t}>0 \text { and } X_{\tau_{0}^{-}(X)}<0 \text { a.s. on }\left\{\tau_{0}^{-}(X)<\infty\right\} \text {. }
$$

For almost every sample path with $\tau_{0}^{-}(X)<\infty$ based on the uniformly convergent coupling of [2, pp.210], we have

$$
\inf _{t \in\left[0, \tau_{0}^{-}(X)\right)} X_{t}^{(n)} \underset{n \uparrow \infty}{\rightarrow} \inf _{t \in\left[0, \tau_{0}^{-}(X)\right)} X_{t} \quad \text { and } \quad X_{\tau_{0}^{-}(X)}^{(n)} \underset{n \uparrow \infty}{\rightarrow} X_{\tau_{0}^{-}(X)},
$$

so that we have

$$
\tau_{0}^{-}(X)=\tau_{0}^{-}\left(X^{(n)}\right) \text { for large } n .
$$

Therefore we have (8) by the dominated convergence theorem. By the strong Markov property, we have

$$
\begin{aligned}
\mathbb{E}_{x}^{X^{(n)}}\left(e^{-q \tau_{0}^{-}} R_{U^{(n)}}^{(q)} f\left(X_{\tau_{0}^{-}}^{(n)}\right)\right) & \\
= & \mathbb{E}_{x}^{X^{(n)}}\left(e^{-q \tau_{0}^{-}} R_{Y^{0(n)}}^{(q)} f\left(X_{\tau_{0}^{-}}^{(n)}\right)\right)+\mathbb{E}_{x}^{X^{(n)}}\left(e^{\Phi_{Y^{(n)}}(q) X^{(n)}\left(\tau_{0}^{-}\right)-q \tau_{0}^{-}}\right) R_{U^{(n)}}^{(q)} f(0) .
\end{aligned}
$$

For the first term we have

$$
\lim _{n \uparrow \infty} \mathbb{E}_{x}^{X^{(n)}}\left(e^{-q \tau_{0}^{-}} R_{Y^{0(n)}}^{(q)} f\left(X_{\tau_{0}^{-}}^{(n)}\right)\right)=\mathbb{E}_{x}^{X}\left(e^{-q \tau_{0}^{-}} R_{Y^{0}}^{(q)} f\left(X_{\tau_{0}^{-}}\right)\right)
$$

where we used the dominated convergence theorem and Lemma 8.3. For the second term we have

$$
\lim _{n \uparrow \infty} \mathbb{E}_{x}^{X^{(n)}}\left(e^{\Phi_{Y^{(n)}}(q) X^{(n)}\left(\tau_{0}^{-}\right)-q \tau_{0}^{-}}\right) R_{U^{(n)}}^{(q)} f(0)=\mathbb{E}_{x}^{X}\left(e^{\Phi_{Y}(q) X\left(\tau_{0}^{-}\right)-q \tau_{0}^{-}}\right) R_{U}^{(q)} f(0)
$$

where we used $\Phi_{Y^{(n)}} \rightarrow \Phi_{Y}$ and (8.4) for $x=0$. The proof is now complete.

For a stochastic process $Z, t>0, x \in \mathbb{R}$ and positive or bounded measurable function $f$, we define

$$
P_{t}^{Z} f(x):=\mathbb{E}_{x}^{Z}\left(f\left(Z_{t}\right)\right)
$$

Theorem 8.5. For all $q>0, t>0$ and $f \in C_{0}$, we have

$$
\begin{aligned}
& R_{U^{(n)}}^{(q)} f \rightarrow R_{U}^{(q)} f \text { uniformly as } n \uparrow \infty, \\
& P_{t}^{U^{(n)}} f \rightarrow P_{t}^{U} f \text { uniformly as } n \uparrow \infty .
\end{aligned}
$$


Proof of Theorems 8.1 and 8.5. From (8.5) we can derive (8.5) by using Theorem 6.4 and [13, Theorem 3.4.2]. Using [6, Theorem 19.25], we can conclude that $\left(U^{(n)}, \mathbb{P}_{x}^{U^{(n)}}\right)$ converges in distribution to $\left(U, \mathbb{P}_{x}^{U}\right)$ for all $x \in \mathbb{R}$.

Let us prove (8.5). We divide the proof of $(8.5)$ into three steps.

Step. 1 Let $k>0$ be a constant. We prove $\left\{\bar{W}_{U^{(n)}}^{(q)}(x)\right\}_{n \in \mathbb{N}}$ is equicontinuous in $x \in$ $[-k, k]$. For this, we prove pointwise convergence $\lim _{n \uparrow \infty} \bar{W}_{U^{(n)}}^{(q)}(x)=\bar{W}_{U}^{(q)}(x)$. Since $\left\{\bar{W}_{U^{(n)}}^{(q)}\right\}_{n \in \mathbb{N}}$ is increasing and continuous by Corollary 6.3, the pointwise convergence implies uniform convergence in $x \in[-k, k]$, thus $\left\{\bar{W}_{U^{(n)}}^{(q)}(x)\right\}_{n \in \mathbb{N}}$ is equicontinuous in $x \in[-k, k]$. The desired convergence is obvious for $x \leq 0$ by the definition of $\bar{W}_{U}^{(q)}(x)$.

For $x>0$, it suffices to show

$$
\lim _{n \uparrow \infty} \mathbb{E}_{0}^{U^{(n)}}\left(e^{-q \tau_{x}^{+}}\right)=\mathbb{E}_{0}^{U}\left(e^{-q \tau_{x}^{+}}\right)
$$

by Corollary 6.3. By the strong Markov property, we have

$$
R_{U^{(n)}}^{(q)} 1_{(-\infty, x)}(0)=\frac{1}{q}\left(1-\mathbb{E}_{0}^{U^{(n)}}\left(e^{-q \tau_{x}^{+}}\right)\right)+\mathbb{E}_{0}^{U^{(n)}}\left(e^{-q \tau_{x}^{+}}\right) R_{U^{(n)}}^{(q)} 1_{(-\infty, x)}(x) .
$$

As $f^{-}:=1_{(-\infty, x)}$ is not continuous, we take bounded continuous functions such that $f_{m}^{-}$and $f_{m}^{+}$such that $f_{m}^{-} \uparrow f^{-}$and $f_{m}^{+} \downarrow f^{+}:=1_{(-\infty, x]}$. Using Theorem [8.4, we have $R_{U^{(n)}}^{(q)} f_{m}^{ \pm} \rightarrow R_{U}^{(q)} f_{m}^{ \pm}$. It is obvious that $R_{U^{(n)}}^{(q)} f^{ \pm} \rightarrow R_{U}^{(q)} f^{ \pm}$. Thus we obtain (8)).

Step.2 We may assume without loss of generality that $\|f\|=1$. Let us prove

$$
R_{U^{(n)}}^{(q)} f(x) \rightarrow R_{U}^{(q)} f(x) \text { uniformly in } x \in[-k, k] .
$$

Since we have the pointwise convergence by Theorem $\mathbf{8 . 4}$, it is sufficient to prove $\left\{R_{U^{(n)}}^{(q)} f\right\}_{n \in \mathbb{N}}$ is equicontinuous. For all $x, y \in \mathbb{R}$ with $x<y$, making a computation similar to $\mathbf{1}$ ) of the proof of Theorem 6.4, we have

$$
\left|R_{U^{(n)}}^{(q)} f(y)-R_{U^{(n)}}^{(q)} f(x)\right| \leq \frac{2}{q}\|f\|\left(1-\frac{\bar{W}_{U^{(n)}}^{(q)}(x)}{\bar{W}_{U^{(n)}}^{(q)}(y)}\right) .
$$

Let $\varepsilon>0$ be a constant. By Step.1 and since $\inf _{n \in \mathbb{N}} \bar{W}_{U^{(n)}}^{(q)}(-k)=\inf _{n \in \mathbb{N}} e^{-\Phi_{Y^{(n)}}(q) k}>0$, we see that there exists $\xi>0$ such that for all $x, y \in[-k, k]$ with $0<y-x<\xi$

$$
\sup _{n \in \mathbb{N}}\left|\bar{W}_{U^{(n)}}^{(q)}(y)-\bar{W}_{U^{(n)}}^{(q)}(x)\right| \leq \varepsilon \inf _{n \in \mathbb{N}} \bar{W}_{U^{(n)}}^{(q)}(-k) .
$$

Then we have

$$
\text { (자) } \leq \frac{2}{q}\|f\| \frac{\varepsilon \inf _{n \in \mathbb{N}} \bar{W}_{U^{(n)}}^{(q)}(-k)}{\bar{W}_{U^{(n)}}^{(q)}(y)} \leq \frac{2}{q}\|f\| \varepsilon \text {, }
$$


where we used the fact that $\bar{W}_{U^{(n)}}^{(q)}$ is increasing. Therefore we conclude that $\left\{R_{U^{(n)}}^{(q)} f\right\}_{n \in \mathbb{N}}$ is equicontinuous.

Step.3 We prove that for any $\varepsilon>0$ there is $k>0$ such that

$$
\sup _{x \in(-\infty,-k) \cup(k, \infty)} \sup _{n \in \mathbb{N}}\left|R_{U^{(n)}}^{(q)} f(x)\right|<\varepsilon .
$$

For all $x<y<0$ we have

$$
\begin{aligned}
\left|R_{U^{(n)}}^{(q)} f(x)\right| & =\left|\mathbb{E}_{x}^{U^{(n)}}\left(\int_{0}^{\tau_{y}^{+}} e^{-q t} f\left(U_{t}^{(n)}\right) d t\right)+\mathbb{E}_{x}^{U^{(n)}}\left(e^{-q \tau_{y}^{+}}\right) R_{U^{(n)}}^{(q)} f(y)\right| \\
& \leq \frac{1}{q} \sup _{z<y}|f(z)|+\frac{1}{q} \sup _{m \in \mathbb{N}} \mathbb{E}_{x}^{Y^{(m)}}\left(e^{-q \tau_{y}^{+}}\right)\|f\| .
\end{aligned}
$$

By the same argument, for all $x>y>0$, we have

$$
\left|R_{U^{(n)}}^{(q)} f(x)\right| \leq \frac{1}{q} \sup _{z>y}|f(z)|+\sup _{m \in \mathbb{N}} \mathbb{E}_{x}^{X^{(m)}}\left(e^{-q \tau_{y}^{-}}\right)\|f\| .
$$

Since $f \in C_{0}$, there exists $k_{1}>0$ such that

$$
\sup _{|z|>k_{1}}|f(z)|<\frac{1}{3} q \varepsilon
$$

Using the uniformly convergence coupling, we have for $x>y>0$

$$
\lim _{n \uparrow \infty} \mathbb{E}_{-x}^{Y^{(n)}}\left(e^{-q \tau_{-y}^{+}}\right)=\mathbb{E}_{-x}^{Y}\left(e^{-q \tau_{-y}^{+}}\right) \quad \text { and } \quad \lim _{n \uparrow \infty} \mathbb{E}_{x}^{X^{(n)}}\left(e^{-q \tau_{y}^{-}}\right)=\mathbb{E}_{x}^{X}\left(e^{-q \tau_{y}^{-}}\right)
$$

and

$$
\lim _{x \uparrow \infty} \mathbb{E}_{-x}^{Y}\left(e^{-q \tau_{-y}^{+}}\right)=0 \quad \text { and } \quad \lim _{x \uparrow \infty} \mathbb{E}_{x}^{X}\left(e^{-q \tau_{y}^{-}}\right)=0 .
$$

By (8), there exists $k_{2}>k_{1}$ such that

$$
\mathbb{E}_{-k_{2}}^{Y}\left(e^{-q \tau_{-k_{1}}^{+}}\right)<\frac{\varepsilon}{3\|f\|} \quad \text { and } \quad \mathbb{E}_{k_{2}}^{X}\left(e^{-q \tau_{k_{1}}^{-}}\right)<\frac{\varepsilon}{3\|f\|}
$$

By (8), there exists $N \in \mathbb{N}$ such that for all $n>N$

$$
\left|\mathbb{E}_{-k_{2}}^{Y^{(n)}}\left(e^{-q \tau_{-k_{1}}^{+}}\right)-\mathbb{E}_{-k_{2}}^{Y}\left(e^{-q \tau_{-k_{1}}^{+}}\right)\right|<\frac{\varepsilon}{3\|f\|}
$$

and

$$
\left|\mathbb{E}_{k_{2}}^{X^{(n)}}\left(e^{-q \tau_{k_{1}}^{+}}\right)-\mathbb{E}_{k_{2}}^{X}\left(e^{-q \tau_{k_{1}}^{+}}\right)\right|<\frac{\varepsilon}{3\|f\|} .
$$

By (8) again, there exists $k_{3}>k_{2}$ such that for all $n \leq N$

$$
\mathbb{E}_{-k_{3}}^{Y^{(n)}}\left(e^{-q \tau_{-k_{1}}^{+}}\right)<\frac{\varepsilon}{3\|f\|} \quad \text { and } \quad \mathbb{E}_{k_{3}}^{X^{(n)}}\left(e^{-q \tau_{k_{1}}^{-}}\right)<\frac{\varepsilon}{3\|f\|}
$$

Thus we obtain

$$
\sup _{n \in \mathbb{N}} \mathbb{E}_{-k_{3}}^{Y^{(n)}}\left(e^{-q \tau_{-k_{1}}^{+}}\right)<\frac{2 \varepsilon}{3\|f\|} \quad \text { and } \quad \sup _{n \in \mathbb{N}} \mathbb{E}_{k_{3}}^{X^{(n)}}\left(e^{-q \tau_{k_{1}}^{-}}\right)<\frac{2 \varepsilon}{3\|f\|} .
$$

By (8), (8), (8) and (8), we obtain (8).

The proof is complete. 


\section{A Constructing generalized a refracted process by excursions}

In this section, we show that we can construct from $n^{U}$ a right-continuous strong Markov processes by means of the excursion theory. We need the following theorem which we state without proof. For $t \geq 0$, we denote $\mathcal{D}_{t}=\sigma(\omega \mapsto \omega(s): s \leq t)$.

Theorem A.1 ([15, Theorem 2]). Let $\left(Z^{0}, \mathbb{P}_{x}^{Z^{0}}\right)$ be a $\mathbb{R}$-valued right-continuous strong Markov process stopped at 0 . Suppose that a $\sigma$-finite measure $n$ on $\mathbb{D}$ satisfies the following conditions:

(i) $n$ is concentrated on $\mathbb{D}^{0}:=\left\{\omega \in \mathbb{D}: \omega(0)=0, T_{0}(\omega)>0, \omega(t)=0\right.$ for $\left.t \geq T_{0}\right\}$.

(ii) $n\left(\mathbb{D}^{0}\right)=\infty$.

(iii) $n\left(1-e^{-T_{0}}\right)<\infty$.

(iv) For all $t>0, A_{1} \in \mathcal{D}_{t}$ with $A_{1} \subset\left\{T_{0}>t\right\}$ and $A_{2} \in \mathcal{B}(\mathbb{D})$,

$$
n\left(A_{1} \cap \theta_{t}^{-1}\left(A_{2}\right)\right)=\int_{A_{1}} \mathbb{P}_{\omega(t)}^{Z^{0}}\left(Z^{0} \in A_{2}\right) n(d \omega),
$$

where $\theta_{t}$ denotes the shift operator.

(v) If a measure $n^{\prime}$ on $\mathbb{D}$ satisfies $n \geq n^{\prime} \geq 0$ and the counterpart of Condition (iv) for $n^{\prime}$, then either $n^{\prime}\left(\mathbb{D}^{0}\right)=0$ or $n^{\prime}\left(\mathbb{D}^{0}\right)=\infty$.

Then there is a right-continuous strong Markov process $Z$ for which $n$ is an excursion measure away from 0 and $\left(Z^{0}, \mathbb{P}_{x}^{Z^{0}}\right)$ is the stopped process.

To construct the strong Markov process $U$ in Section 5, we need to check that $U^{0}$ is a right-continuous strong Markov process and that $n^{U}$ satisfies conditions of Theorem A.1.

Lemma A.2. The stopped process $\left(U^{0}, \mathbb{P}_{x}^{U^{0}}\right)$ has the Markov property.

Proof. It is obvious that $\left(U^{0}, \mathbb{P}_{x}^{U^{0}}\right)=\left(Y^{0}, \mathbb{P}_{x}^{0}\right)$ for $x<0$ satisfies the Markov property. We thus need to prove that $\left(U^{0}, \mathbb{P}_{x}^{U^{0}}\right)$ satisfies the Markov property for $x>0$. Let $A_{1} \in \mathcal{D}_{t}$ with $A_{1} \subset\left\{T_{0}>t\right\}$ and $A_{2} \in \mathcal{B}(\mathbb{D})$. We write $A=A_{1} \cap \theta_{t}^{-1}\left(A_{2}\right)$. By the definition of $\mathbb{P}_{x}^{U^{0}}$, we have

$$
\begin{aligned}
\mathbb{P}_{x}^{U^{0}}\left(U^{0} \in A\right)= & \mathbb{E}_{x}^{X}\left(\left.\mathbb{P}_{y}^{Y^{0}}\left(w \circ Y^{0} \in A\right)\right|_{\substack{y=X\left(\tau_{0}^{-}\right) \\
w=(X(s))_{s<\tau_{0}^{-}}}} ; \tau_{0}^{-} \leq t\right) \\
& +\mathbb{E}_{x}^{X}\left(\left.\mathbb{P}_{y}^{Y^{0}}\left(w \circ Y^{0} \in A\right)\right|_{\substack{y=X\left(\tau_{0}^{-}\right) \\
w=(X(s))_{s<\tau_{0}^{-}}}} ; t>\tau_{0}^{-}\right),
\end{aligned}
$$


where $w \circ w^{\prime}$ denotes the concatenation of a path $w=\left(w_{s}\right)_{s<s_{0}}$ of finite length $s_{0}$ and a path $w^{\prime}=\left(w_{s}^{\prime}\right)_{s \geq 0}$ of infinite length:

$$
\left(w \circ w^{\prime}\right)_{s}= \begin{cases}w_{s} & s<s_{0}, \\ w_{s-s_{0}}^{\prime} & s \geq s_{0} .\end{cases}
$$

By the Markov property of $Y^{0}$, we have

$$
\begin{aligned}
& \mathbb{E}_{x}^{X}\left(\left.\mathbb{P}_{y}^{Y^{0}}\left(w \circ Y^{0} \in A\right)\right|_{\substack{y=X\left(\tau_{0}^{-}\right) \\
w=(X(s))_{s<\tau_{0}^{-}}}} ; \tau_{0}^{-} \leq t\right) \\
= & \mathbb{E}_{x}^{X}\left(\left.\mathbb{P}_{y}^{Y^{0}}\left(w \circ Y^{0} \in A_{1},\left(Y_{s}^{0}\right)_{s \geq t-u} \in A_{2}\right)\right|_{\substack{y=X_{u} \\
w=(X(s))_{s<u} \\
u=\tau_{0}^{-}}} ; \tau_{0}^{-} \leq t\right) \\
= & \mathbb{E}_{x}^{X}\left(\left.\mathbb{E}_{y}^{Y^{0}}\left(\left.1_{\left\{w \circ Y^{0} \in A_{1}\right\}} \mathbb{P}_{y^{\prime}}^{Y^{0}}\left(Y^{0} \in A_{2}\right)\right|_{y^{\prime}=Y_{t-u}^{0}}\right)\right|_{\substack{y=X_{u} \\
w=(X(s))_{s<u} \\
u=\tau_{0}^{-}}} ; \tau_{0}^{-} \leq t\right) \\
= & \mathbb{E}_{x}^{U}\left(\left.1_{\left\{U \in A_{1}\right\}} \mathbb{P}_{y}^{U^{0}}\left(U^{0} \in A_{2}\right)\right|_{y=U_{t}} ; \tau_{0}^{-} \leq t\right) .
\end{aligned}
$$

We can do a similar argument for (A). So we obtain

$$
\mathbb{P}_{x}^{U^{0}}\left(U^{0} \in A\right)=\int_{A_{1}} \mathbb{P}_{\omega(t)}^{U^{0}}\left(U^{0} \in A_{2}\right) \mathbb{P}_{x}^{U^{0}}\left(U^{0} \in d \omega\right)
$$

The proof is complete.

Lemma A.3. The stopped process $U^{0}$ has the strong Markov property.

Proof. Fix $t>0$. By the proof of [4, Theorem 1 of Section 2.3], it is sufficient to prove that $x \mapsto \mathbb{E}_{x}^{U^{0}}\left(f\left(U_{t}^{0}\right)\right)$ is continuous for all bounded continuous function $f$ with $f(0)=0$. Continuity at $x<0$ is obvious, by the Feller property of $Y^{0}$. Left-continuity at $x=0$ is also obvious. Right-continuity at $x=0$ follows from the fact that $\mathbb{P}_{y}^{U^{0}}\left(T_{0} \in \cdot\right) \underset{y \rightarrow 0}{\rightarrow} \delta_{0}$. Let us consider continuity at $x>0$.

$$
\begin{aligned}
\mathbb{E}_{y}^{U^{0}}\left(f\left(U_{t}^{0}\right)\right)= & \mathbb{E}_{y}^{U^{0}}\left(f\left(U_{t}^{0}\right) ; \tau_{0}^{-} \wedge t<T_{x}\right)+\mathbb{E}_{y}^{X}\left(f\left(X_{t}\right) ; T_{x} \leq t<\tau_{0}^{-}\right) \\
& +\mathbb{E}_{y}^{X}\left(\left.\mathbb{E}_{y^{\prime}}^{Y^{0}}\left(f\left(Y_{t-u}^{0}\right)\right)\right|_{\substack{y^{\prime}=X(u) \\
u=\tau_{0}^{-}}} ; T_{x} \leq \tau_{0}^{-} \leq t\right) .
\end{aligned}
$$

Note that we have $\mathbb{P}_{0}^{X}\left(\lim _{y \rightarrow 0} T_{y}=0\right)=1$ by the assumption that $X$ is spectrally negative and of bounded variation. Since $X$ and $Y^{0}$ have càdlàg paths, we have the following 
identities:

$$
\begin{aligned}
& \mathbb{E}_{y}^{U^{0}}\left(f\left(U_{t}^{0}\right) ; \tau_{0}^{-} \wedge t<T_{x}\right) \leq\|f\| \mathbb{P}_{0}^{X}\left(\tau_{-\frac{x}{2}}^{-}<T_{x-y}\right) \underset{y \rightarrow x}{\rightarrow} 0, \\
& \mathbb{E}_{y}^{X}\left(f\left(X_{t}\right) ; T_{x} \leq t<\tau_{0}^{-}\right)=\mathbb{E}_{y}^{X}\left(\left.\mathbb{E}_{x}^{X}\left(f\left(X_{t-u}\right) ; t<\tau_{0}^{-}\right)\right|_{u=T_{x}} ; T_{x} \leq t \wedge \tau_{0}^{-}\right) \\
& \mathbb{E}_{x}^{X}\left(f\left(X_{t}\right) ; t<\tau_{0}^{-}\right), \\
& \quad \mathbb{E}_{y}^{X}\left(\left.\mathbb{E}_{y^{\prime}}^{Y^{0}}\left(f\left(Y_{t-u}^{0}\right)\right)\right|_{\substack{y^{\prime}=X(u) \\
u=\tau_{0}^{-}}} ; T_{x} \leq \tau_{0}^{-} \leq t\right) \\
& \quad \mathbb{E}_{y}^{X}\left(\left.\mathbb{E}_{x}^{X}\left(\left.\mathbb{E}_{y^{\prime}}^{Y^{0}}\left(f\left(Y_{t-u-v}^{0}\right)\right)\right|_{\substack{y^{\prime}=X(u) \\
u=\tau_{0}^{-}}} ; \tau_{0}^{-} \leq t\right)\right|_{\substack { v=T_{x} \\
\begin{subarray}{c}{\rightarrow \rightarrow x{ v = T _ { x } \\
\begin{subarray} { c } { \rightarrow \rightarrow x } }\end{subarray}} ; T_{x} \leq \tau_{0}^{-} \wedge t\right) \\
& \mathbb{E}_{x}^{X}\left(\left.\mathbb{E}_{y^{\prime}}^{Y^{0}}\left(f\left(Y_{t-u}^{0}\right)\right)\right|_{\substack{y^{\prime}=X(u) \\
u=\tau_{0}^{-}}} ; \tau_{0}^{-} \leq t\right) .
\end{aligned}
$$

The proof is now complete.

Lemma A.4. The measure $n=n^{U}$ satisfies Conditions (ii), (iii), (iii), (iv) and (四) in Theorem A.1.

Proof. It is obvious by definition that $n^{U}$ satisfies (ii) and (iii).

Let us prove (iii). By the definition of $n^{U}$ and by (2), we have

$$
\begin{aligned}
n^{U}\left(1-e^{-T_{0}}\right) & =n^{X}\left(1-e^{-\tau_{0}^{-}} \mathbb{E}_{X_{\tau_{0}^{-}}^{Y}}\left(e^{-\tau_{0}^{+}}\right) 1_{\left\{\tau_{0}^{-}<\infty\right\}}\right) \\
& =n^{X}\left(1-e^{-\tau_{0}^{-}} e^{\Phi_{Y}(1) X_{\tau_{0}^{-}}} 1_{\left\{\tau_{0}^{-}<\infty\right\}}\right) .
\end{aligned}
$$

We let $q^{\prime}=1 \vee \inf \left\{q>0: \Phi_{X}(q)>\Phi_{Y}(1)\right\}$. Since $n^{X}\left(1-e^{-q^{\prime} T_{0}}\right)$ is finite, we obtain

$$
\begin{aligned}
(\underline{\mathrm{A}}) & \leq n^{X}\left(1-e^{-q^{\prime} \tau_{0}^{-}} e^{\Phi_{X}\left(q^{\prime}\right) X_{\tau_{0}^{-}}} 1_{\left\{\tau_{0}^{-}<\infty\right\}}\right) \\
& =n^{X}\left(1-e^{-q^{\prime} \tau_{0}^{-}} \mathbb{E}_{X_{\tau_{0}^{-}}^{X}}^{X}\left(e^{-q^{\prime} \tau_{0}^{+}}\right) 1_{\left\{\tau_{0}^{-}<\infty\right\}}\right)<\infty .
\end{aligned}
$$

The proof of (iv) is the same as that of the Markov property of $\left(U^{0}, \mathbb{P}_{x}^{U^{0}}\right)$ for $x>0$ in Lemma A.2.

Let us prove (可). We define the $\sigma$-finite measure $n^{\prime \prime}$ by

$$
n^{\prime \prime}\left(F\left(\left(U_{t}\right)_{t<\tau_{0}^{-}},\left(U_{t+\tau_{0}^{-}}\right)_{t \geq 0}\right)\right)=n^{\prime}\left(\left.\mathbb{E}_{y}^{X^{0}}\left(F\left(w,\left(X_{t}^{0}\right)_{t \geq 0}\right)\right)\right|_{\substack{y=U\left(\tau_{0}^{-}\right) \\ w=(U(t)) \\ t<\tau_{0}^{-}}}\right)
$$


for all non-negative measurable functional $F$. Then $n^{\prime \prime}$ satisfies the Markov property for $\left\{\mathbb{P}_{x}^{X^{0}}\right\}_{x \in \mathbb{R} \backslash\{0\}}$. By the definition of $n^{U}$, we have $n^{X} \geq n^{\prime \prime} \geq 0$. By [15, Proposition 1], $n^{X}$ satisfies Condition ( $(\mathbb{\nabla})$ and we obtain either $n^{\prime \prime}\left(\mathbb{D}_{0}\right)=0$ or $n^{\prime \prime}\left(\mathbb{D}_{0}\right)=\infty$, which yields we have either $n^{\prime}\left(\mathbb{D}_{0}\right)=0$ or $n^{\prime}\left(\mathbb{D}_{0}\right)=\infty$.

The proof is complete.

Acknowledgments. The authors were supported by JSPS-MAEDI Sakura program. The second author was supported by MEXT KAKENHI grant no.'s 26800058 and 15 H03624.

\section{References}

[1] F. Avram, J. L. Peréz and K. Yamazaki. Spectrally negative Lévy processes with Parisian reflection below and classical reflection above. (English summary) Stochastic Process. Appl. 128 (2018), no. 1, 255-290.

[2] J. Bertoin. Lévy processes. Cambridge Tracts in Mathematics, 121. Cambridge University Press, Cambridge, 1996. x+265 pp.

[3] Z.-Q. Chen and M. Fukushima. One-point reflection. Stochastic Process. Appl. 125 (2015), no. 4, 1368-1393.

[4] K. L. Chung and J. B. Walsh. Markov processes, Brownian motion, and time symmetry. Second edition. Grundlehren der Mathematischen Wissenschaften [Fundamental Principles of Mathematical Sciences], 249. Springer, New York, 2005. xii+431 pp.

[5] R. A. Doney. Some Excursion Calculations for Spectrally One-sided Lévy Processes. Séminaire de Probabilités XXXVIII, 5-15, Lecture Notes in Mathematics., 1857, Springer, Berlin, 2005.

[6] O. Kallenberg. Foundations of Modern Probability. Second edition. Probability and its Applications (New York). Springer-Verlag, New York, 2002. xx+638 pp.

[7] A. Kuznetsov, A. E. Kyprianou and V. Rivero. The theory of scale functions for spectrally negative Lévy processes. In, Lévy matters II, 97-186, Lecture Notes in Math., 2061, Springer, Heidelberg, 2012.

[8] A. E. Kyprianou. Gerber-Shiu risk theory. European Actuarial Academy (EAA) Series. Springer, Cham, 2013. viii+93 pp.

[9] A. E. Kyprianou. Fluctuations of Lévy Processes with Applications. Introductory lectures. Second edition. Universitext. Springer, Heidelberg, 2014. xviii+455 pp.

[10] A. E. Kyprianou and R. L. Loeffen. Refracted Lévy processes. Ann. Inst. Henri Poincaré Probab. Stat. 46 (2010), no. 1, 24-44. 
[11] J. C. Pardo, J. L. Pérez, and V. Rivero. The excursion measure away from zero for spectrally negative Lévy processes. Ann. Inst. Henri Poincaré Probab. Stat., to appear.

[12] J. C. Pardo, J. L. Pérez, and V. Rivero. Lévy insurance risk processes with parisian type severity of debt. arXiv:1507.07255, July 2015.

[13] A. Pazy. Semigroups of linear operators and applications to partial differential equations. Applied Mathematical Sciences, 44. Springer-Verlag, New York, 1983. viii+279 pp.

[14] L. C. G. Rogers and D. Williams. David Diffusions, Markov processes, and martingales. Vol. 2. Itô calculus. Reprint of the second (1994) edition. Cambridge Mathematical Library. Cambridge University Press, Cambridge, 2000. xiv+480 pp.

[15] T. S. Salisbury. On the Itô excursion process. Probab. Theory Related Fields 73 (1986), no. 3, 319-350.

[16] K. Yano and Y. Yano. On h-transforms of one-dimensional diffusions stopped upon hitting zero. In memoriam Marc Yor-Séminaire de Probabilités XLVII, 127-156, Lecture Notes in Math., 2137, Springer, Cham, 2015. 